\title{
Asymmetric Attosecond Photoionization in Molecular Shape Resonance
}

\author{
Xiaochun Gong $\odot,{ }^{1,4}$ Wenyu Jiang, ${ }^{1}$ Jihong Tong, ${ }^{1}$ Junjie Qiang, ${ }^{1}$ Peifen Lu ${ }^{1}$ Hongcheng Ni@, ${ }^{1,4, *}$ \\ Robert Lucchese, ${ }^{2}$ Kiyoshi Ueda $\odot^{1,3, \uparrow}$ and Jian $\mathrm{Wu}^{1,4,5, \$}$ \\ ${ }^{1}$ State Key Laboratory of Precision Spectroscopy, East China Normal University, Shanghai 200241, China \\ ${ }^{2}$ Lawrence Berkeley National Laboratory, Berkeley, California 94720, USA \\ ${ }^{3}$ Department of Chemistry, Tohoku University, Sendai 980-8578, Japan \\ ${ }^{4}$ Collaborative Innovation Center of Extreme Optics, Shanxi University, Taiyuan, Shanxi 030006, China \\ ${ }^{5}$ CAS Center for Excellence in Ultra-intense Laser Science, Shanghai 201800, China
}

(Received 15 April 2021; accepted 8 November 2021; published 4 January 2022)

\begin{abstract}
A shape resonance emerges during the light absorption in many molecules with a gigantic burst amplitude and a lifetime of hundreds of attoseconds. Recent advances in attosecond metrology revealed the attosecond lifetime of the shape resonance. For a heteronuclear molecule, the asymmetric initial state and landscape of the molecular potential would lead to an asymmetric shape resonance, whose effect, however, has not been characterized yet. Here, we employ an attosecond interferometer to investigate the molecularframe photoionization time delay in the vicinity of the shape resonance of the NO molecule. Driven by photons with energy ranging from $23.8 \mathrm{eV}$ to $36.5 \mathrm{eV}$, a 150 attosecond difference in the time delay is observed between photoemission from the N/O end. Our quantum scattering theoretical simulations reproduce well our experimental findings. It illustrates that the asymmetric time delay originates from the interference between resonant and nonresonant photoionization pathways.
\end{abstract}

DOI: 10.1103/PhysRevX.12.011002

\section{INTRODUCTION}

A shape resonance arises from a transient state in which an electron is trapped temporally inside a potential barrier [1-6] or a cage [7-9] that emerges during light absorption, with a gigantic burst amplitude, of most molecules in the gas and condensed phases. It can be related to a quasibound state with a well-defined angular momentum, as sketched in Fig. 1, where the trapped electron tunnels through a centrifugal potential barrier into the continuum, within a few electron volts in the energy domain [10-14] and a few hundred attoseconds in the time domain [15-18], serving as a prototype to investigate the energy, temporal, and spatial properties of the transiently trapped electron wave packet. With the advances of attosecond metrology, e.g., the attosecond streak camera [19-21] and the technique of reconstruction of attosecond harmonic beating by interference of two-photon transitions (RABBITT) [22], timing the electron wave-packet dynamics in photoionization via

\footnotetext{
*hcni@lps.ecnu.edu.cn

†kiyoshi.ueda@tohoku.ac.jp

\#jwu@phy.ecnu.edu.cn
}

Published by the American Physical Society under the terms of the Creative Commons Attribution 4.0 International license. Further distribution of this work must maintain attribution to the author(s) and the published article's title, journal citation, and DOI.
Subject Areas: Atomic and Molecular Physics, Chemical Physics, Optics the shape resonance can be achieved on the attosecond timescale.

Photoionization time delay, i.e., the time delay of the photoelectron wave packet caused by the scattering potential $[21,23]$, is theoretically modeled as the Eisenbud-WignerSmith (EWS) delay $\tau_{\text {EWS }}[24,25]$. The photoionization, often considered as a half-scattering process, is sensitive to the dynamical centrifugal potential that leads to the shape resonance. The time delay of the tunneled photoelectron wave packet in the vicinity of the shape resonance, which corresponds to the lifetime of the shape resonance, has been experimentally reported recently $[13,14,17,18]$. However, these time-delay measurements were either averaged over the molecular orientation [17,18], distinguished solely between parallel and perpendicular cases [26], or reconstructed from static photoelectron angular distributions $[13,14]$. The angle-resolved timing of the shape resonance, particularly the asymmetric angle-resolved electron scattering dynamics in the asymmetric molecular potential landscape as illustrated in Fig. 1, has not been characterized so far.

Molecular-frame photoelectron angular distribution $[27,28]$ is a sensitive probe of the symmetry and shape of the continuum wave packets, as well as the photoelectron scattering process in the asymmetric molecular potential. In this article, we experimentally investigate the novel asymmetric attosecond photoemission dynamics in the heteronuclear NO molecule in its molecular frame by 


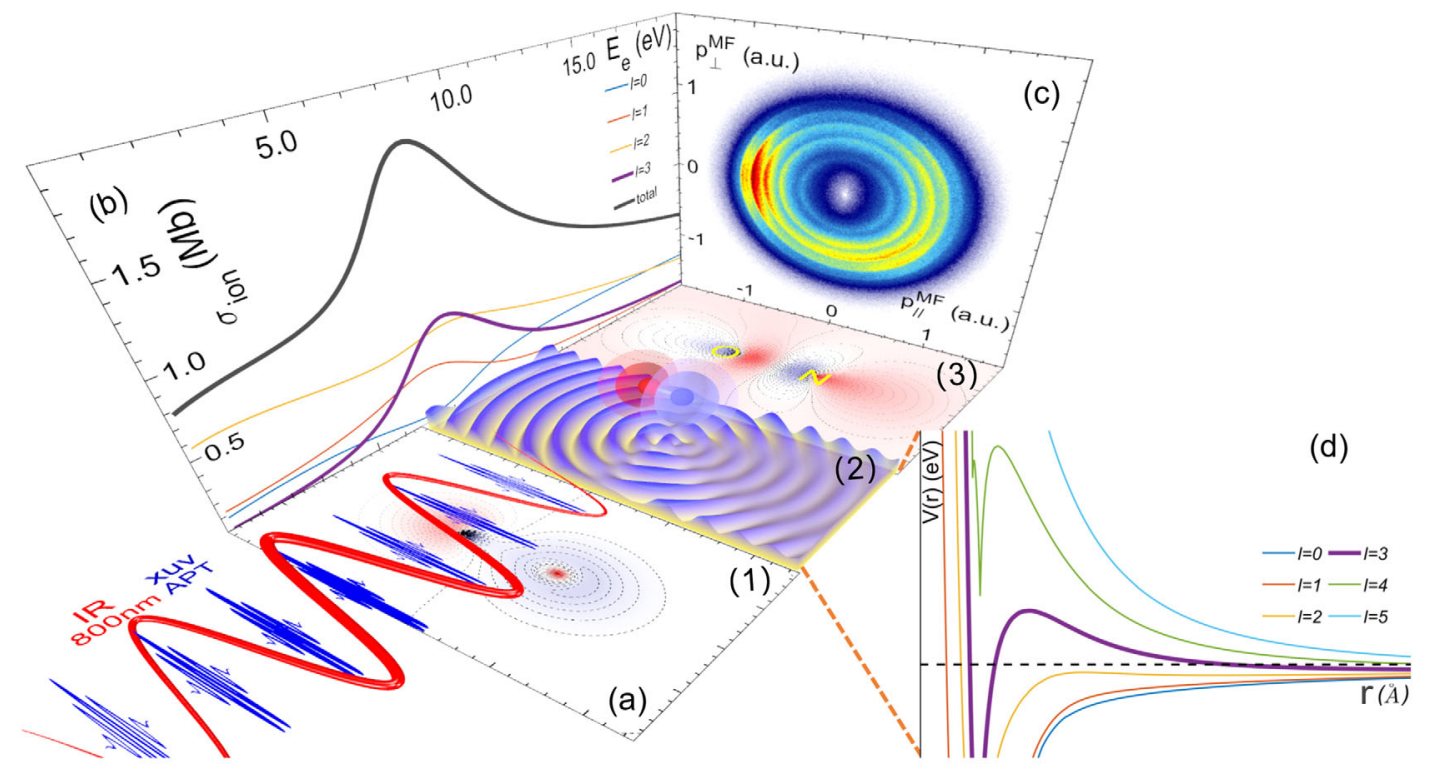

FIG. 1. Asymmetric electron tunneling dynamics. (a) Schematic diagram of the molecular orientation-dependent photoemission dynamics from the dynamical centrifugal potential barrier in the perimeter of the NO molecule via the inner-valence electron removal of $4 \sigma$. The NO molecule is an open-shell molecule with the electronic ground state of $X^{2} \Pi$ under the configuration of $(1 \sigma)^{2}(2 \sigma)^{2}(3 \sigma)^{2}(4 \sigma)^{2}(5 \sigma)^{2}(1 \pi)^{4}(2 \pi)^{1}$ with $\mathrm{C}_{\infty v}$ symmetry. The expected shape resonance occurs during the photoionization upon electron removal from the $4 \sigma$ inner-valence shell generating a $c^{3} \Pi \mathrm{NO}^{+}$ionic state $\left(I_{p}=21.7 \mathrm{eV}\right)$, giving rise to the $\mathrm{N}^{+}+\mathrm{O}$ nuclear fragments in the end. The $4 \sigma$ electron orbital [panel (1)] and resonant continuum wave [panel (3)] are aligned along the internuclear axis, where the nuclear positions are labeled by the yellow character [panel (3)], and the positive and negative signs of the electron wave function are labeled by the red and blue areas. The wave function [panel (2)] is a schematic sketch of the partial-wave interference arising from the $\mathrm{N}$ and $\mathrm{O}$ atomic centers. The asymmetric photoelectron emission was clocked using a phase-locked XUV-APT and NIR attosecond coincidence interferometer in the molecular frame. (b) Total photoionization cross section as well as that for individual partial waves in the vicinity of the shape resonance. The enhancement of the cross section due to the shape resonance appears mainly in the $f$ partial wave ( $l=3$, purple line). (c) Momentum diagram $p_{\|}^{\mathrm{MF}}$ vs $p_{\perp}^{\mathrm{MF}}$ showing the photoelectron momentum distribution in the molecular frame with the $\mathrm{N}$ and $\mathrm{O}$ atoms aligning along the axis of $p_{\|}^{\mathrm{MF}}$, where $p_{\perp}^{\mathrm{MF}}$ is perpendicular to the molecular bond axis, in the dipole plane perpendicular to the light propagation axis. In this panel, the polarization vector is averaged over all directions in the dipole plane. (d) Atomic potential together with the centrifugal potential from the high $l$ scattering channels, forming a potential barrier that transiently traps the electron, leading to the observed shape resonance as quantified by the partial-wave-resolved cross sections in (b) $(l=3$, purple line $)$.

employing the advanced attosecond coincidence interferometer with the advantage of three-dimensional momentum recording feasibility from the cold-target recoil-ion momentum spectroscopy (COLTRIMS) [29] and attosecond time resolution from the protocol of RABBITT. We carry out a typical RABBITT measurement by ionizing the NO molecule with a phase-locked near-infrared (NIR) femtosecond laser pulse and an extreme ultraviolet attosecond pulse train (XUV-APT) [30] covering the photon energy of the main high-order harmonics from the 15th $(\mathrm{M} 15,23.85 \mathrm{eV})$ to the 23rd order (M23, $36.57 \mathrm{eV})$. We measure an angle- and energy-resolved photoionization time delay in the vicinity of the shape resonance and find a large time-delay difference of about 150 as between photoelectrons emitted from the $\mathrm{N} / \mathrm{O}$ end of the $\mathrm{NO}$ molecule. Our single-center $a b$ initio quantum scattering calculation demonstrates that the molecular orientationdependent asymmetry in the time delay can be ascribed to the coherent partial-wave interference between the nonresonant and the resonant photoionization pathways caused by the dynamical centrifugal potential barrier during the electron emission process. A further two-center treatment provides a complementary view of the asymmetric time delay. In particular, we find that the asymmetry is largely induced by the asymmetric final scattering state, while the asymmetry in the initial state accentuates the difference and shifts the asymmetry to the energy region observed.

\section{RESULTS AND DISCUSSIONS}

In the present study, we focus on the photoionization of the NO molecule upon electron removal from the $4 \sigma$ inner-valence shell generating a $c^{3} \Pi \mathrm{NO}^{+}$ionic state $\left(I_{p}=21.7 \mathrm{eV}\right)$ via the parallel transition, giving rise to the $\mathrm{N}^{+}+\mathrm{O}$ nuclear fragments along the light polarization vector in the end, as illustrated in Fig. 2(a). Figure 2(b) depicts the key results, the measured photoionization 

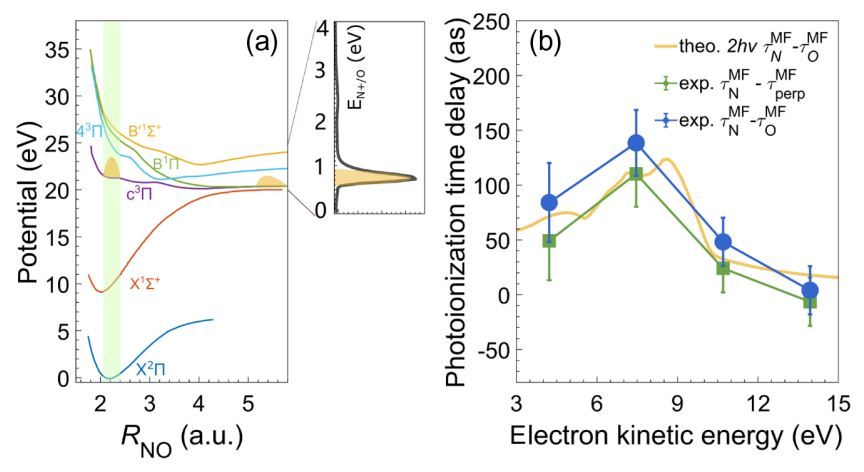

FIG. 2. Molecular orientation-dependent asymmetric photoemission time delays. (a) Potential energy curves of the relevant electronic states during the photoionization of the NO molecule [31]. The green area indicates the Franck-Condon region, and the dissociation pathway from the $c^{3} \Pi$ state is highlighted via a yellow area. The inset shows the kinetic energy distributions of $\mathrm{N}^{+}$and $\mathrm{O}$ in the dissociation channel of $\mathrm{NO}^{+}$. (b) Experimentally measured photoemission time-delay difference as a function of electron kinetic energy in the vicinity of the shape resonance of the NO molecule. The blue dots show the delay difference when the photoelectron emits to the $\mathrm{N}$ end, $\tau_{\mathrm{N}}^{\mathrm{MF}}$, and to the $\mathrm{O}$ end, $\tau_{\mathrm{O}}^{\mathrm{MF}}$, of the NO molecule for parallel transitions in which the $\mathrm{N}^{+}$ions are ejected along the light polarization vector [see Fig. 1(a)]. For comparison, the green dots show the difference between $\tau_{\mathrm{N}}^{\mathrm{MF}}$ and the delay when the photoelectron is emitted perpendicularly to the $\mathrm{N}^{+}$momentum $\tau_{\perp}^{\mathrm{MF}}$ for the perpendicular transitions in which the $\mathrm{N}^{+}$ions are ejected perpendicularly to the light polarization axis. The $a b$ initio two-photon ionization time delay is shown as the yellow curve. The details of the molecular-frame coordinate rotation and the selections of the data are shown in the Appendix B. The error bars indicate the standard deviation.

time-delay difference between photoelectron emission to the $\mathrm{N} / \mathrm{O}$ end $\left(\tau_{\mathrm{N}-\mathrm{O}}^{\mathrm{MF}}=\tau_{\mathrm{N}}^{\mathrm{MF}}-\tau_{\mathrm{O}}^{\mathrm{MF}}\right)$ of the NO molecule in the molecular frame (MF). A large variation of the timedelay difference is observed in the vicinity of the shape resonance, and a maximal $\tau_{\mathrm{N}-\mathrm{O}}^{\mathrm{MF}}=150$ as is found near the peak at a photoelectron energy of $E_{e} \approx 7.0 \mathrm{eV}$.

As schematically shown in Fig. 1, the photoemission delay across the shape resonance of the NO molecule is clocked using the RABBITT technique [22]. It is one of the most widely used attosecond metrologies $[20,21,32]$ to achieve accurate attosecond timing of photoelectron emission dynamics, and it has demonstrated a prominent attosecond probing capability in the time and frequency domains [33] with targets ranging from isolated atoms [30,34-38] and molecules $[15,17,18,26,39-42]$ to complex clusters [43] and even liquids [44] and solids [45-48]. Here, we construct the attosecond coincidence interferometer based on a hollow-core waveguide high-harmonic generation source (XUUS4.0) [49]. The NIR pulse $(1.0 \mathrm{~mJ}, 25 \mathrm{fs})$ is split into two beams with equal intensities by a beam splitter (50:50). One NIR beam is focused into a waveguide filled with argon gas (gas pressure of about 40 mbar) to generate the XUV-APT (see Appendix A), and the other beam serves as the probe pulse. In the end, the phase-locked XUV-APT and a weak NIR pulse [30,43] are focused onto a supersonic gas jet in the ultrahigh vacuum chamber of COLTRIMS, where the 3D momenta of the photoionization and photodissociation-induced electron and ion fragments [29] are measured in coincidence as a function of the pump-probe delay between the XUV-APT and NIR pulses. The intensity of the NIR laser field is estimated to be $2.0 \times 10^{12} \mathrm{~W} / \mathrm{cm}^{2}$ [50].

In our COLTRIMS experiment, we measure the molecular axis orientation based on the axial recoil approximation [51]. Depending on the specific electronic states excited by a laser pulse, the molecule may rotate, leading to the breakdown of the axial recoil approximation. The axial recoil approximation indeed holds in the dissociation channel along the $c^{3} \Pi$ state we are concerned with here (see Appendix B). The photoelectron momentum angular resolution in the molecular frame is estimated to be $3^{\circ}$, accounting for the angular resolutions of the ion and electron detectors and the minor possible rotation of the molecular axis during the molecule's fast dissociation.

Figures 3(a), 3(b), 3(d), and 3(e) show the experimentally measured photoelectron energy spectra of the $1 \mathrm{~s}$ orbital of the He atom $\left(I_{p}=24.58 \mathrm{eV}\right)$ and the $4 \sigma$ orbital $\left(I_{p}=21.7 \mathrm{eV}\right)$ of the NO molecule as a function of the relative time delay $\tau$ between the XUV-APT and NIR, showing up as an oscillation at the frequency of $2 \omega$, with $\omega$ the angular frequency of the NIR dressing field. As shown in Figs. 3(a) and 3(d), the absorption and/or emission of a NIR photon from two neighboring main harmonic peaks generate a photoelectron interference sideband (SB) between these two quantum pathways, serving as an interferometer for timing the photoelectron emission dynamics. The SB oscillation follows as $A_{\mathrm{SB}} \propto A_{2 \omega} \cos \left(2 \omega \tau+\phi_{0}\right)$, where $\phi_{0}=\Delta \phi_{\mathrm{XUV}}+\Delta \phi_{\mathrm{sys}}^{\mathrm{SB}}$, and $\Delta \phi_{\mathrm{XUV}}$ denotes the optical dispersion of the driven XUV-APT and $\Delta \phi_{\mathrm{sys}}^{\mathrm{SB}}=\Delta \phi_{\mathrm{sys}}^{\mathrm{mol}}+$ $\Delta \phi_{\mathrm{cc}}$ encodes the system-specific scattering phase underlying XUV + IR two-photon ionization dynamics, including the molecular photoionization phase shift $\left(\Delta \phi_{\mathrm{sys}}^{\mathrm{mol}}\right)[24,25]$ and the additional continuum-continuum phase shift $\Delta \phi_{\mathrm{cc}}$ induced by the NIR pulse in the Coulomb field [52] (see Appendix A). Figures 3(c) and 3(d) show the measured SB oscillation phase in $\mathrm{He}^{+}$and $\mathrm{NO}^{+}$. Analogously to the time delay in the atomic photoionization process, SB time delay is obtained from the SB oscillation phase as $\tau^{\mathrm{SB}}=\phi_{0} / 2 \omega=$ $\tau_{\mathrm{sys}}^{\mathrm{SB}}+\tau_{\mathrm{XUV}}$, where the group delay of the XUV-APT is independent of the target, be it an atom or a molecule.

To retrieve the effective photoelectron emission time delay leading to the $\mathrm{c}^{3} \Pi$ state of $\mathrm{NO}^{+}\left(\tau_{\mathrm{N}^{+}, \mathrm{O}}^{\text {abs }}\right)$ in the vicinity of the shape resonance, we use the helium atom as an external reference to calibrate the photoemission time delay in the NO molecule since the photoemission time delay of helium $\left(\tau_{\mathrm{He}^{+}}\right)$is well established [53-56]. It can be used to cancel out the chirp of the XUV-APT in our experiments 

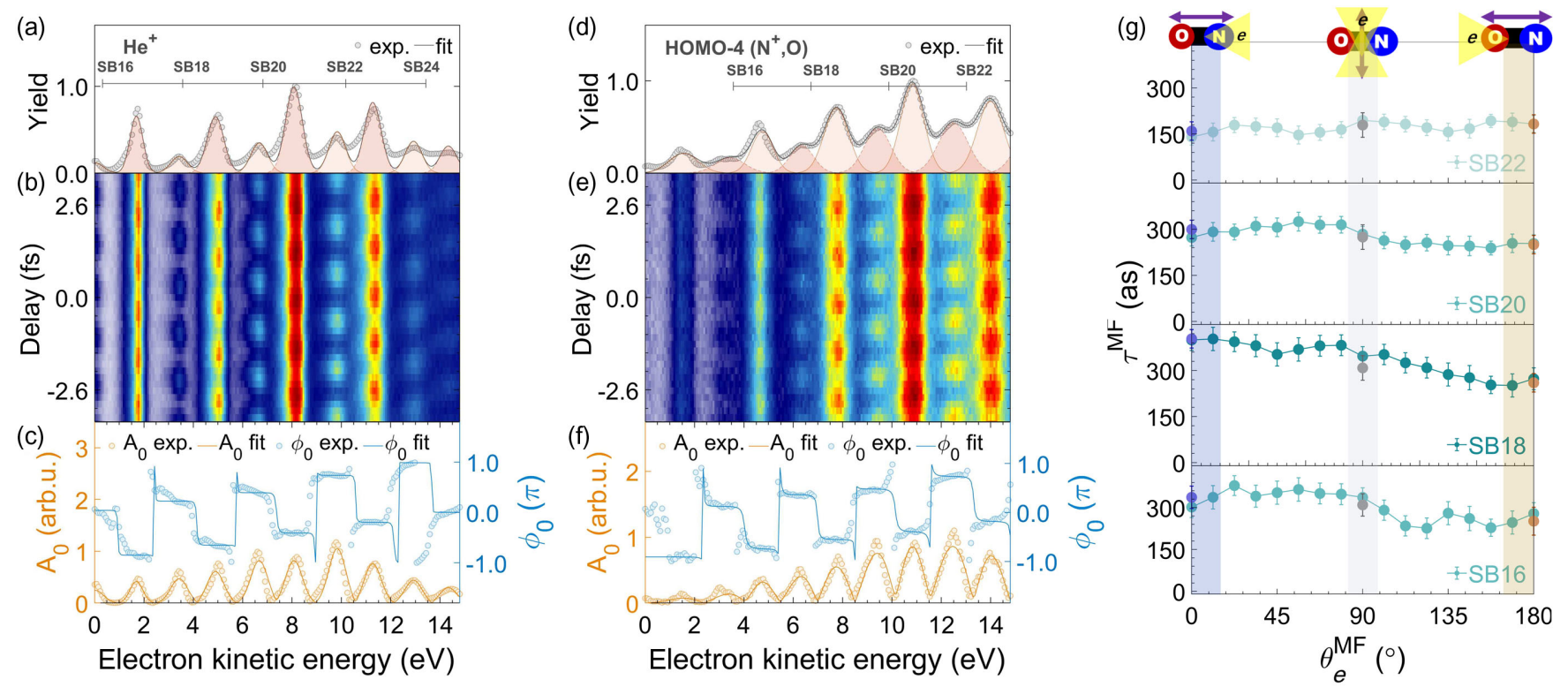

FIG. 3. Attosecond photoelectron spectroscopy. (a) Time-averaged photoelectron energy spectrum (PES) from the helium atom under the radiation of the XUV-APT and NIR. The light red areas are the numerical fits using Gaussian distributions. (b) RABBITT trace of helium, i.e., the PES as a function of the pump-probe delay between the XUV-APT and NIR. (c) Oscillation amplitude and phase of the PES. The fitted results are shown as solid lines using a complex fitting method. (d)-(f) Same as (a)-(c) but for the photoionization from the $4 \sigma$ inner-valence shell of the NO molecule. (g) Energy-resolved photoelectron emission time delay as a function of the photoelectron emission angle $\left(\theta_{e}^{\mathrm{MF}}\right)$ in the molecular frame extracted from SB16 to SB22. The blue (orange) dots at $\theta_{e}^{\mathrm{MF}}=0^{\circ}\left(180^{\circ}\right)$ show the time delay of the photoelectron emitting to the $\mathrm{N}$ atom (to the $\mathrm{O}$ atom) integrated over a cone of $30^{\circ}$ of the $\theta_{e}^{\mathrm{MF}}$ under the parallel transition conditions (the $\mathrm{N}^{+}$ions along the polarization direction of the XUV-APT with a detection cone of $30^{\circ}$ ). The gray dots at $\theta_{e}^{\mathrm{MF}}=90^{\circ}$ show the photoelectron time delays under the perpendicular transitions (the $\mathrm{N}^{+}$ion perpendicular to the polarization direction of the XUV-APT). The cyan dots show the angle-resolved time delays, without transition selections, i.e., with the light polarization averaged over all directions in the dipole plane. The blue and orange shaded areas indicate photoemission to the $\mathrm{N}$ end and to the $\mathrm{O}$ end under parallel transitions, respectively. The insets schematically show the geometry of the molecular orientation and the polarization direction of the XUV-APT, and the yellow shaded areas represent the photoemission direction with respect to the orientation of the NO molecule. The error bars show the standard deviation.

and the continuum-continuum transition delay. The absolute time delay of the NO molecule is then defined as $\tau_{\exp }^{\mathrm{NO}}=\tau_{\mathrm{N}^{+}, \mathrm{O}}^{\mathrm{SB}}-\tau_{\mathrm{He}^{+}}^{\mathrm{SB}}+\tau_{\mathrm{He}^{+}}+\delta \tau_{\mathrm{cc}^{+}}^{\mathrm{He}^{+}-\mathrm{N}^{+}, \mathrm{O}}$ (see Appendix $\mathrm{A}$ ). To investigate the spatial property of the photoelectron emission dynamics in the vicinity of the shape resonance, we transform the measured momentum vectors of the photoelectron $\boldsymbol{P}_{e}$ into the molecular frame based on ionmomentum vectors of $\boldsymbol{P}_{\mathrm{N}^{+}}$measured in coincidence, which are generated from the fast dissociation pathway from the $\mathrm{c}^{3} \Pi$ state of $\mathrm{NO}^{+}[6]$. Figure 3(g) shows the energy- and angle-resolved photoelectron emission time delay in the molecular frame. Here, the data dot at $\theta_{e}^{\mathrm{MF}}=0^{\circ}$ corresponds to the case of emission to the $\mathrm{N}$ end with a time delay of $\tau_{\mathrm{N}}^{\mathrm{MF}}$ under the parallel transition. Likewise, the one at $\theta_{e}^{\mathrm{MF}}=180^{\circ}$ stands for the opposite case of emission to the $\mathrm{O}$ end with a delay of $\tau_{\mathrm{O}}^{\mathrm{MF}}$. An asymmetric electron emission time-delay distribution could be well observed in the molecular frame. The data dot at $\theta_{e}^{\mathrm{MF}}=90^{\circ}$ corresponds to electron emission perpendicular to the molecular axis, for the perpendicular $\pi$ transition, with a weak energy dependence in the present photon energy range. It is dominated by a $\pi$ partial wave in the continuum, whose lobes are aligned along the light polarization axis. The asymmetric photoelectron emission time delay between parallel and perpendicular transitions, $\tau_{\mathrm{N}}^{\mathrm{MF}}-\tau_{\perp}^{\mathrm{MF}}$, is also shown in Fig. 2(b) (labeled as green dots).

The shape resonance is caused by the transient trapping of the electron inside the barrier formed by the centrifugal potential and is intrinsically linked to the phase accumulated by the departing photoelectron. To gain further insights into the nature of the shape resonance and the resulting asymmetric emission direction-dependent delay, we carry out a quantum scattering calculation with a onecenter expansion of scattering partial waves from the mass center of the NO molecule [57-60] (see Appendix D). In the following, we distinguish the partial wave of a singlephoton $(1 h \nu)$ process to reach the main peak, denoted by the small $l$, and that of the two-photon $(2 h \nu)$ process to reach the SB as in the RABBITT scheme, denoted by the capital $L$. Figure 1(b) depicts the cross sections of the scattering amplitude for different $l$ partial-wave channels under the single XUV photoionization of the $4 \sigma$ electron. We notice that the $l=3(f)$ partial-wave cross section 
exhibits enhancement at the shape resonance, though the cross sections of other partial waves also show some minor modulations in its vicinity. Referring to the partial-waveresolved centrifugal potential barrier depicted in Fig. 1(d), we notice that only the $l=3$ channel has a proper effective trapping barrier. For channels with lower $l$, the barrier is not high enough to temporally trap the departing electron. For channels with higher $l$, the barrier $(46 \mathrm{eV}$ for $l=4)$ is too high for an initial quasibound state to get populated in the first place. It is this particular $l=3$ channel that contributes most prominently to the observed shape resonance, which we define as the resonant channel in the single-photon absorption process. This resonant channel further maps to $L=2$ and $L=4$ channels following the XUV-NIR two-photon transition process since $L=l \pm 1$. Shown in Fig. 4(a) are the scattering time delays of different $L$ partialwave channels following the XUV + NIR two-photon parallel transition for a continuum wave with $A_{1}$ symmetry (see Appendix D). Evidently, a large delay variation occurs for the $L=2$ (yellow line) and $L=4$ (red line) channels in the energy region of the shape resonance, corresponding to the large cross section of the $l=3$ resonance channel (purple line) shown in Fig. 1(b). In contrast, the time delays for other nonresonant channels are flatter.

Given the flexibility of the scattering partial-wave analysis, we show, in addition, the theoretical calculation with resonant and nonresonant channels separately. In Fig. 4(b), we show the measured (red round dots) molecular time delay $\left(\tau_{\mathrm{exp}}^{\mathrm{NO}}\right)$ emitted from the $4 \sigma$ orbital, which is integrated over the photoelectron emission angle of $\theta_{e}^{\mathrm{MF}}$, and the photoionization time delays of the nonresonant channel (superposition of $L=0,1,3$ partial waves, black line) and the resonant channel (superposition of $L=2,4$ partial waves, red line), and their coherent superposition (yellow line). We find a maximal time delay around the position of the shape resonance. The good consistency between theoretical and experimental results reveals that it is the $f$-wave $(l=3)$ channel that leads to the increase in the time delay around the shape resonance because switching it off would effectively eliminate the associated centrifugal barrier, which transiently traps the electron for both single-photon and two-photon cases.

The present scattering partial-wave analysis is also in line with the measured relative time delay with the electron emitting to different directions in the molecular frame. Figure 4(c) shows the asymmetric photoionization time delay between the emission to $\mathrm{N}$ and $\mathrm{O}$ ends. Here, both the nonresonant and resonant channels show only a weak asymmetry. The observed giant photoemission delay difference from different ends of the molecule results from the coherent superposition of the resonant and nonresonant channels. Shown also in Fig. 2(b) as the yellow line is the relative delay in the one-center scattering model when the electron emits to the $\mathrm{N}$ and the $\mathrm{O}$ ends in the molecular frame, in agreement with experimental results.

In order to pin down the origin of the asymmetric time delay in the spatial domain, we further carry out a complementary analytical two-center multiple scattering analysis (see Appendix E). With such a model, we can selectively make both the initial and final state symmetric or asymmetric. Shown in Fig. 5 is such a study of the influence of the initial- and final-state symmetry on the angle-averaged time delay [Fig. 5(a)] and the asymmetric time delay between emission to $\mathrm{N}$ and $\mathrm{O}$ [Fig. 5(b)],
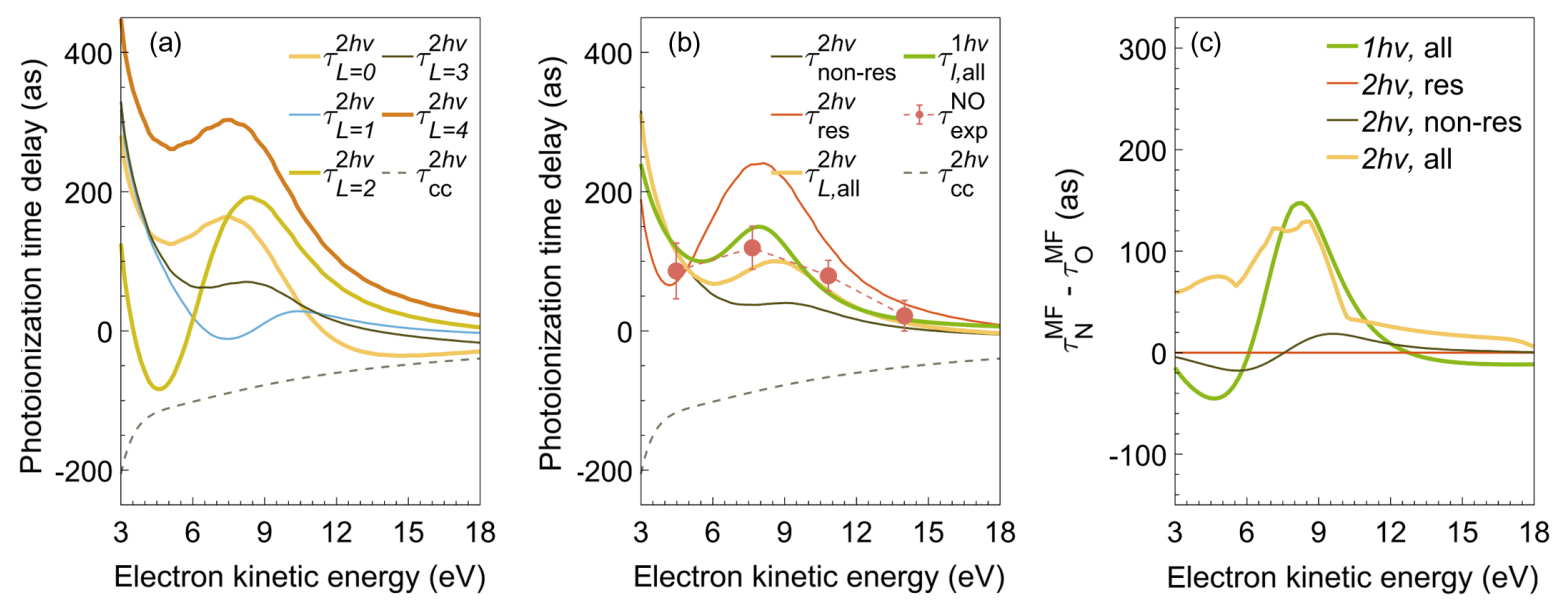

FIG. 4. Scattering partial-wave analysis. (a) Photoionization time delays for different partial-wave channels in the vicinity of the shape resonance via the photoionization from the $4 \sigma$ inner-valence shell of NO. The contributions of the $f$ wave $(l=3)$ are shown in the $L=2$ and $L=4$ channels. The dashed line shows the continuum-continuum transition time delay. (b) Photoionization time delay of the resonant $(L=2,4$, red line) and nonresonant $(L=0,1,3$, black line) channels as well as the delay from their coherent superposition (all $L$, yellow line). Red round dots show the experimentally measured photoionization time delays $\left(\tau_{\exp }^{\mathrm{NO}}\right)$ that are integrated over all photoelectron emission angles in the parallel transition, and the green curve shows the photoionization time delay in the one-photon ionization. (c) Resonant (red line) and nonresonant (black line) asymmetric photoionization time delay in the molecular frame. The light-yellow and light-green curves show the calculated two-photon $(2 h \nu)$ and one-photon $(1 h \nu)$ asymmetric ionization time delays. 

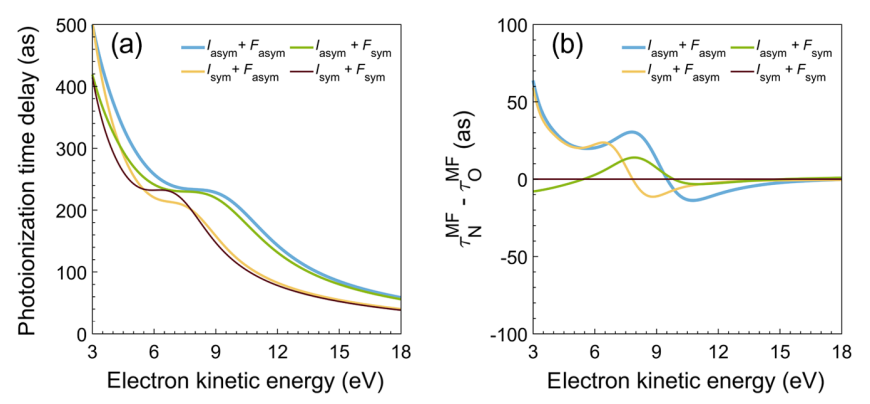

FIG. 5. Asymmetry analysis of the molecular-frame photoionization time delay. (a) Angle-averaged photoionization time delays calculated via the two-center multiple scattering model. The relative asymmetries between the initial and final states are both characterized. (b) Same as (a) but for the relative photoionization time delay between $\mathrm{N} / \mathrm{O}$ ends $\tau_{\mathrm{N}}^{\mathrm{MF}}-\tau_{\mathrm{O}}^{\mathrm{MF}}$.

where $I_{\text {sym/asym }}$ denotes the case when the initial state is symmetric/asymmetric and $F_{\text {sym/asym }}$ stands for the case where the final state is symmetric/asymmetric. From Fig. 5(a), it is clear that all four combinations of the symmetric/asymmetric initial/final states give rise to the shape resonance, while the location of the resonance depends more on the initial state than on the final state. From Fig. 5(b), we find that the asymmetry in both the initial and final states plays an important role in inducing the asymmetric time delay when the electron emits to different sides. When the initial and final states are both symmetric, the asymmetric time delay vanishes, as expected. In particular, the asymmetric potential landscape of the final scattering state mainly leads to the prominent observed asymmetric time delay, and the asymmetry in the initial state accentuates such asymmetric delay to the energy region observed in the experiment. We note that the one-center (frequency-domain interpretation) and two-center (spatialdomain interpretation) pictures are the complementary faces of the asymmetric photoemission time delay, and which picture to use is only a matter of taste and convenience.

\section{CONCLUSION}

To conclude, our measurements and simulations reveal the asymmetric character of the transiently trapped outgoing photoelectron wave packet in the vicinity of the shape resonance of the NO molecule in the molecular frame. Our results directly probe the quantum partial-wave interference effects in the asymmetric angle-resolved electron scattering dynamics from the dynamical centrifugal potential barriers. Our simulations show that the observed shape resonance is created by the $f$-wave quasibound state. A one-center approach and a complementary two-center approach have been employed to explain the asymmetric angle-dependent time delays. In the one-center approach, the asymmetric photoionization time delay between the $\mathrm{N}$ end and $\mathrm{O}$ end is caused by the interference between the resonant and the nonresonant partial-wave channels. In the two-center picture, on the other hand, the asymmetric delay arises mostly from the asymmetric molecular potential landscape. Our work relies on the validity of axial recoil approximation, which is satisfied in the studied KER region of the NO molecule. We note that it may not hold equally well for the $\mathrm{CO}$ molecule under our experimental conditions (see Appendix C). The resonant trapping of the electron wave packet by a centrifugal potential is also common for giant resonances that appear in heavy atoms and shape resonances in molecules with a high degree of symmetry such as $\mathrm{SF}_{6}$, while these examples do not show the asymmetric time delay caused by the asymmetric scattering potentials studied here. Despite being demonstrated in heteronuclear diatomic molecules, our methods and findings are applicable to photoemission dynamics via any resonance in various molecules, surfaces, and interfaces that have asymmetric potentials. Our approach opens a new perspective on exploring the attosecond photoelectron dynamics in complex systems [61,62], and on investigating the time-resolved quantum dynamics in solutions, complex materials, and biologic tissues.

\section{ACKNOWLEDGMENTS}

We acknowledge Denis Jelovina, Trung Tran Luu, Denitsa R. Baykusheva, Joachim Burgdörfer, and Hans Jakob Wörner for helpful conversations on the photoionization time-delay calculations. We acknowledge support from the National Key R\&D Program of China (Grants No. 2018YFA0306303 and No. 2019YFA0308300), the National Natural Science Foundation of China (Grants No. 61690224, No. 11974114, No. 11804098, No. 11834004, No. 11904103, No. 91950203, No. 11621404, and No. 12122404), the Shanghai Science and Technology Commission (Grants No. 19JC1412200, No. 18ZR1412400, No. 21ZR1420100, and No. 19560745900), the 111 Project of China (Grant No. B12024), and the Fundamental Research Funds for the Central Universities. Numerical simulations were, in part, performed on the ECNU Multifunctional Platform for Innovation (001). We have also used the resources of the National Energy Research Scientific Computing Center, a DOE Office of Science User Facility, and the Lawrencium computational cluster resource provided by the IT Division at Lawrence Berkeley National Laboratory. The theoretical research performed at Lawrence Berkeley National Laboratory was supported by the DOE, Office of Science, Office of Basic Energy Sciences, Chemical Sciences, Geosciences, and Biosciences Division, under Contract No. DE-AC02-05CH11231.

\section{APPENDIX A: MATERIALS AND METHODS}

\section{XUV-APT source}

The tabletop extreme ultraviolet (XUV) attosecond light source is based on the high-order harmonic generation 
(HHG) scheme in a capillary waveguide [49], which is filled with argon gas at a pressure of 40 mbar. The capillary waveguide has a total length of $6 \mathrm{~cm}$ and an inner diameter of $150 \mu \mathrm{m}$. The HHG process [63,64] is driven by a multipass amplified Ti:sapphire laser system (RAEA), which delivers near-infrared (NIR) femtosecond laser pulses with $1.6 \mathrm{~mJ}$ energy at a $10-\mathrm{kHz}$ repetition rate, a central wavelength of $780 \mathrm{~nm}$ and a pulse duration of $28 \mathrm{fs}$. A coaxial 200-nm aluminum foil on a quartz ring is placed before a rare gold-coated toroidal mirror $(f=50 \mathrm{~cm})$ to gate the XUV spectrum and block the residual IR pulse copropagating with the XUV beam. The generated XUV attosecond pulse train (XUV-APT) is recombined with the remaining part of the IR beam after the toroidal mirror via a central holed silver mirror to constitute a Mach-Zehnder interferometer. The relative time delay between the XUVAPT and NIR pulse is controlled via a combined motor stage including a high-precision direct-current motor working on a femtosecond timescale and a piezoelectric motor on an attosecond timescale. The relative delay between the XUV-APT and NIR pulse is actively controlled via a close-loop feedback-control algorithm with a time jitter of 22 attoseconds $[30,43,65]$. The XUV-APT covers the high-harmonic orders from the 15th $(\mathrm{M} 15,23.85 \mathrm{eV})$ to 23rd (M23, $36.57 \mathrm{eV})$. Based on the measured rare gas RABBITT spectrum (argon atom), the electric field of the XUV-APT is reconstructed via the reconstruction approach of the frequency-resolved optical gating for complete reconstruction of attosecond bursts (FROG$\mathrm{CRAB}$ ) through the iterative algorithm of principal component generalized projection algorithms (PCGPA) $[66,67]$. Figures 6(a) and 6(b) show the measured and reconstructed RABBITT trace, respectively. As plotted in Fig. 6(c), a full width at half maximum at 295 attoseconds is estimated from the reconstruction.

\section{Phase reconstruction}

Compared to atomic systems, the measurement of molecular photoionization time delays typically faces the challenge of spectral overlap [68]. This is because the XUV-APT ionizes electrons out of several possible orbitals, which leads to spectral overlap between the photoelectron MB spectra created by different harmonic orders and the
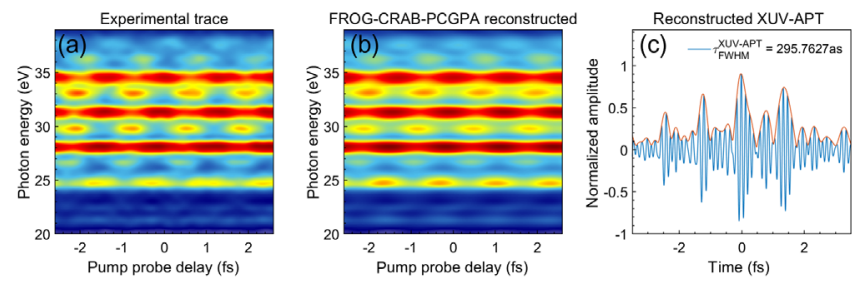

FIG. 6. Pulse characterization of the XUV-APT. (a) Measured RABBITT spectrum of the argon atom. (b) Reconstructed RABBITT spectrum via FROG-CRAB-PCGPA. (c) Reconstructed electric field of the XUV-APT (blue line) and its envelope (red line).
SB spectra. To extract the photoionization time delays from spectrally overlapping attosecond photoelectron spectra, we use the complex-valued principal component analysis as described in detail in Refs. [43,68,69]. In the first step, we use multiple Gaussian functions to reproduce the XUV + NIR photoelectron spectrum with respect to the ionization potential of the molecule and the XUV photon energy. Next, a fast-Fourier transformation (FFT) is carried out line by line on the photoelectron spectra along the time-delay axis in the experimentally measured photoelectron spectrum, as done in Fig. 6(a), and the resulting band in the complex-valued FFT at the $2 \omega$ angular frequency is fitted by multiplying each Gaussian component obtained in the $\mathrm{XUV}+\mathrm{NIR}$ fit with a complex amplitude $e^{z_{j}}$,

$$
I_{\mathrm{fit}}(E)=\sum_{j} p_{j}(E) e^{z_{j}}
$$

where $p_{j}(E)$ is the Gaussian fit for photoelectron $b$ and $j$, and $I_{\text {fit }}(E)$ is the photoelectron yield along the pumpprobe time-delay axis at a certain photoelectron kinetic energy $E$. This complex number $z_{j}=a_{j}+i b_{j}$ simultaneously accounts for the sideband specific delay $\tau_{j}=$ $-b_{j} /(2 \omega)$ and a finite modulation contrast when $\left|e^{a_{j}}\right|<1$.

\section{Molecular photoionization time-delay reconstruction approach}

The atomic photoionization time delay is interpreted in terms of the Eisenbud-Wigner-Smith (EWS) delay $\tau_{\text {EWS }}$ $[24,25]$ to the outgoing electron wave scattering in the external potential as compared to the same wave passing through a free space. The two-photon XUV + NIR transition-induced photoionization time delay can be decomposed into two items, $\tau=\tau_{\text {sys }}+\tau_{\mathrm{cc}}$, where $\tau_{\mathrm{sys}}$ is accumulated from the photoionization process in the absence of the phase-locked XUV-APT and NIR laser field and the $\tau_{\mathrm{cc}}$ is induced by the continuum-continuum transition through the Coulomb-laser coupling. As illustrated in Refs. [52,70-72], the $\tau_{\mathrm{cc}}$ term is only sensitive to the frequency of the probe field (NIR), the Coulomb field of

TABLE I. Correction of the photoionization time delay of NO molecule. The theoretically calculated helium and argon atomic effective photoionization time delays are used as a reference to calibrate the chirp of the XUV-APT and the continuumcontinuum transition time delay.

\begin{tabular}{lcccc}
\hline \hline SB orders & $\mathbf{1 6}$ & $\mathbf{1 8}$ & $\mathbf{2 0}$ & $\mathbf{2 2}$ \\
\hline$\tau_{\mathrm{EWS}}^{\mathrm{He}^{+}}$(as) & 33.60 & 15.70 & 11.30 & 8.80 \\
$\phi_{\mathrm{exp}}^{\mathrm{He}}(\boldsymbol{\pi})$ & 0.0373 & 0.2308 & 0.4007 & 0.7379 \\
$\delta \tau_{\mathrm{XUV}}$ (as) & 138.1 & 255.8 & 484.5 & 655.2 \\
$\delta \tau_{\mathrm{cc}}^{\mathrm{NO}-\mathrm{He}}$ (as) & 60 & 35 & 20.3 & 15 \\
$\tau_{\mathrm{EWS}}^{\mathrm{Ar}}$ (as) & 92 & 80 & 64 & 44 \\
$\delta \tau_{\mathrm{cc}}^{\mathrm{Ar}}-\mathrm{He}$ (as) & 25 & 20 & 11 & 9.0 \\
\hline \hline
\end{tabular}




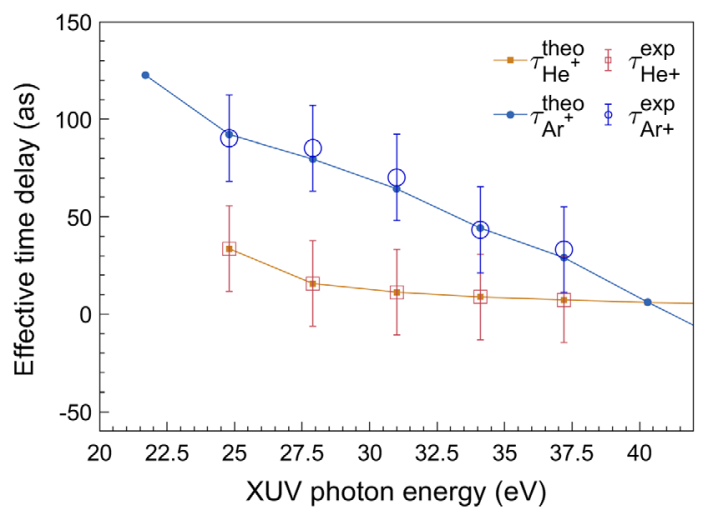

FIG. 7. Corrected atomic photoionization time delay. The open red squares and blue circles show the angle-integrated photoionization time delay of helium and argon, respectively. The delays are corrected based on Table I. The solid dotted lines are taken from Refs. [53].

the parent ion, and the final kinetic energy of the released photoelectron. As shown in Fig. 4(c) in the main text, we use the continuum-continuum delays from exact calculations in hydrogen to correct the ionization potential difference between the helium atom and the $4 \sigma$ orbital of the NO molecule from SB16 to SB22, as shown in Table I. The $\tau_{\mathrm{EWS}}^{\mathrm{He}^{+}}$is the theoretically calculated result as shown in Ref. [53]. The $\phi_{\mathrm{exp}}^{\mathrm{He}^{+}}$shows the measured sideband phases of a $1 \mathrm{~s}$ electron ionized from the helium atom, and $\delta \tau_{\mathrm{XUV}}$ indicates the XUV pulse-related dispersion calculated via the difference $\left(\phi_{\mathrm{exp}}^{\mathrm{He}^{+}} / 2 \omega_{\mathrm{NIR}}\right)-\tau_{\mathrm{EWS}}^{\mathrm{He}^{+}}$. The $\delta \tau_{\mathrm{cc}}^{\mathrm{NO}-\mathrm{He}}$ is calculated via the difference of $\tau_{\mathrm{cc}}^{I_{p}=21.7 \mathrm{eV}}-\tau_{\mathrm{cc}}^{I_{p}=24.58 \mathrm{eV}}$. As in the correction in the NO molecule, as shown in Fig. 7, here we also take into account the calibration for the measured photoionization time delay of the argon atom.

\section{APPENDIX B: NUCLEAR ROTATION EFFECT OF NO}

\section{Measurement of electron and ion momenta in coincidence}

The phase-locked XUV-APT and NIR pulses are collinearly focused onto the supersonic gas jet in a COLTRIMS $[29,73,74]$ spectrometer. The electrons and ions created by XUV photoionization are guided by a weak homogeneous electric field $\left(3.50 \mathrm{~V} \cdot \mathrm{cm}^{-1}\right)$ and a homogeneous magnetic field $(6.80 \mathrm{G})$ towards two time- and position-sensitive detectors at the opposite end of the spectrometer. For the electrons, the length of the extraction region is about $8 \mathrm{~cm}$, followed by a 16-cm field-free region. A homogeneous magnetic field is applied over the center regions of the spectrometer by a set of Helmholtz coils with a diameter of $1.5 \mathrm{~m}$. By setting all the electrons in a single rotation wiggle, we could achieve a full threedimensional momentum electron-ion coincidence measurement in a $4 \pi$ solid angle. In our experiments, $x$ corresponds to the direction of light propagation, $y$ is along the direction of the supersonic gas jet, and $z$ is along the time-offlight of the ions. The photoelectron kinetic energy is calibrated via the XUV photoionization spectrum of the $3 p$ electron of an argon atom.

Figure 8(a) shows the measured joint energy spectrum between ions and electrons in the dissociative ionization pathway of NO. The photoelectrons removed from the $4 \sigma$ orbital correlate with the ionic kinetic energy of $\mathrm{N}^{+}\left(E_{\mathrm{ion}}^{\mathrm{N}}\right)$ around $0.45 \mathrm{eV}$ [6], corresponding to the narrow half-moon structures in the momentum spectrum as plotted in Fig. 8(b). To single out the photoelectron effect from other electronic states, we set a gate of $0.4 \mathrm{eV}<E_{\text {ion }}^{\mathrm{N}}<0.5 \mathrm{eV}$ to the $\mathrm{N}^{+}$ion signals generated by the following dissociative ionization process as shown in Eq. (A1), where the parallel and perpendicular transitions could generate continuum electrons with $\sigma$ and $\pi$ symmetry, respectively:

$$
\begin{aligned}
& \mathrm{NO}\left(X^{2} \Pi,(4 \sigma)^{2}(5 \sigma)^{2}(1 \pi)^{4}(2 \pi)^{1}\right)+h \nu \\
& \quad \rightarrow \mathrm{NO}^{+}\left(c^{3} \Pi,(4 \sigma)^{-1}\right)+\mathrm{e}(\sigma) \text { or } \mathrm{e}(\pi) \\
& \quad \rightarrow \mathrm{N}^{+}+\mathrm{O}+\mathrm{e} .
\end{aligned}
$$

Figure 9 shows the molecular-frame photoelectron angular distributions corresponding to the photoionization process (S1). The molecular-frame coordinates are determined by using the vector correlations of the light prorogation direction, the ion vector of $\mathrm{N}^{+}$, and the vector of the coincidentally measured electron. After the coordinate rotation, the photoelectrons are projected to the plane perpendicular to the light propagation direction (dipole plane), $p_{\|}^{\mathrm{MF}}$ vs $p_{\perp}^{\mathrm{MF}}$, where the vector of $\mathrm{N}^{+}$is oriented along the direction of the $p_{\|}^{\mathrm{MF}}$. Figure 9(a) shows the photoelectron angular distribution without any ionmomentum gating before the coordinate transformation. Figures 9(b) and 9(c) show the photoelectron angular distribution with an ion-momentum gating of $\mathrm{N}^{+}$with a cone of $30^{\circ}$ along or perpendicular to the XUV polarization axis, respectively, which corresponds to the parallel and
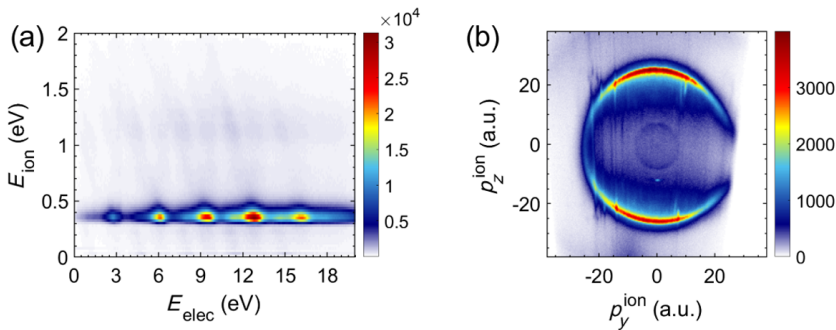

FIG. 8. Dissociative ionization pathway of the NO molecule. (a) Joint energy spectrum of ions and electrons, $E_{\text {ion }}$ vs $E_{\text {elec }}$. The $E_{\text {ion }}$ indicates the kinetic energy of the $N^{+}$. (b) Ionmomentum distribution in the laser polarization plane, where the polarization direction of the XUV and NIR pulses is polarized along the $z$ axis. 

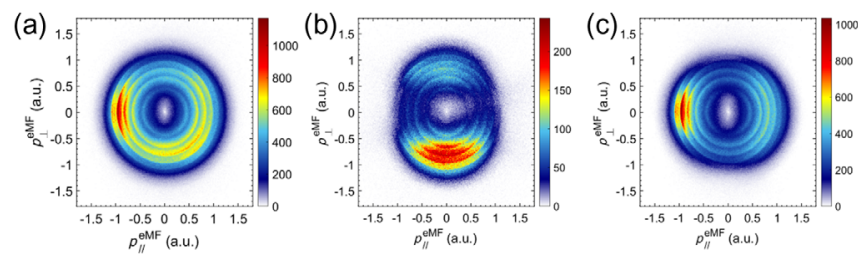

FIG. 9. Measured molecular-frame photoelectron angular distribution of the NO molecule. (a) Nuclear-orientation-integrated photoelectron angular distribution in the molecular frame with a polarization vector averaged over all directions in the dipole plane perpendicular to the light propagation axis, where the photoelectron momentum $p_{\|}^{\mathrm{MF}}$ is defined to be parallel with the molecular bond axis, and the $p_{\perp}^{\mathrm{MF}}$ is perpendicular to the nuclear bond axis, in the dipole plane, corresponding to the direction of the $\pi$ preferred transition. (b,c) Same as (a) but for the ionmomentum gate as (b) the perpendicular transition and (c) the parallel transition.

perpendicular dipole transitions at the instant of the single XUV photon absorption. The asymmetric photoelectron angular distribution of Fig. 9(c) is ascribed to the gigantic electron ionization in the vicinity of the shape resonance. In addition, as shown in Fig. 8(b), the asymmetric momentum distributions along $p_{\perp}^{\mathrm{MF}}$ originate from the missing ion signals around $p_{y}^{\text {ion }} \approx 20$ a.u. Figure 10 presents the ionfragment angular distribution of $\mathrm{N}^{+}$released from the electronic state of $c^{3} \Pi\left(0.4 \mathrm{eV}<E_{\text {ion }}^{\mathrm{N}}<0.5 \mathrm{eV}\right)$ integrated over the XUV photon energy. It exhibits a strong molecular axial dissociation dependence. By using the function of $I\left(\theta_{\mathrm{N}}\right)=I_{0}\left\{1+\beta_{2} P_{2}\left[\cos \left(\theta_{\mathrm{N}}\right)\right]\right\}$ to fit the ionic angular distribution as a function of the photon energy in Fig. 11(a), we obtain the asymmetric parameter $\beta_{2}$ of the

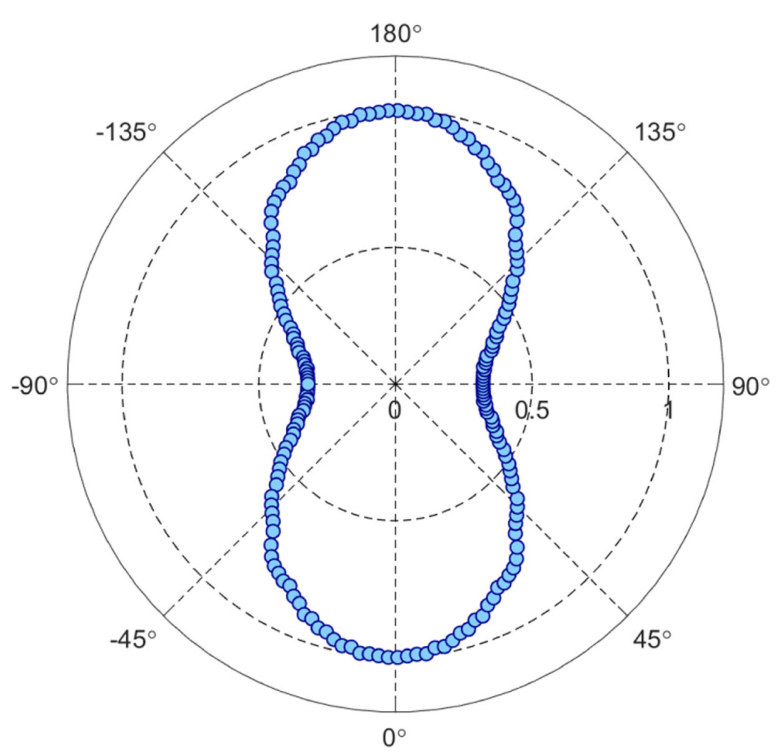

FIG. 10. Ion-momentum angular distribution of $\mathrm{N}^{+}$released from the $c^{3} \Pi$ electronic state. The results follow the reflection symmetry along the polarization axis of the XUV-APT.

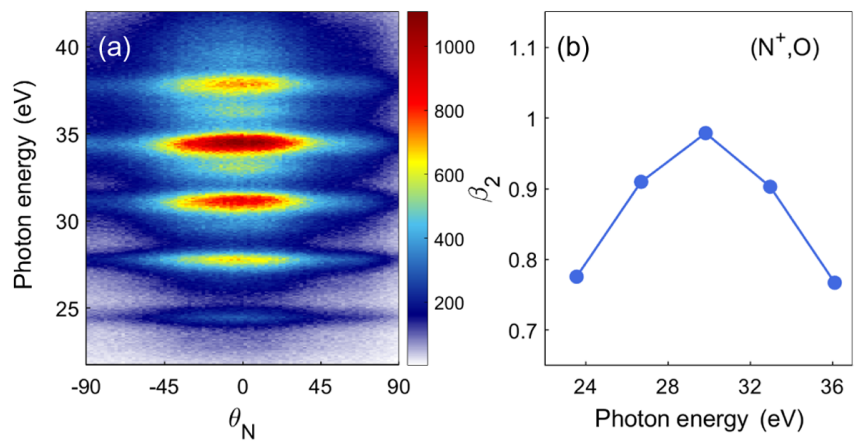

FIG. 11. (a) Ion angular distribution of $\mathrm{N}^{+}$versus the XUV photon energy. (b) Fitted asymmetric parameters $\beta_{2}$ of the $\mathrm{N}^{+}$ion as a function of the XUV photon energy.

$\mathrm{N}^{+}$ion fragments, where $\theta_{\mathrm{N}}$ is the angle between the light polarization vector and the emission direction of the fragment ion in the dipole plane, $P_{2}$ is the second-order Legendre polynomial, and $\beta_{2}$ is the asymmetric parameter [51]. Figure 11(b) shows the results at each XUV-photon energy position from M15 to M21. If the axial recoil approximation is valid, then $\beta_{2}=2$ for the parallel transition, $\beta_{2}=-1$ for the perpendicular transition, and the value of $\beta_{2}$ between -1 and 2 gives the intensity ratio of the parallel and perpendicular transitions. If the axial recoil approximation breaks down, the $\beta_{2}$ value approaches zero. The increase of the $\beta_{2}$ value at about $30 \mathrm{eV}$ therefore corresponds to the enhancement of the parallel transition from the $4 \sigma$ to $\sigma$ continuum via the $\sigma^{*}$ shape resonance and illustrates that the axial recoil approximation holds for this dissociation channel, as also shown in Ref. [6].

\section{One-dimensional quantum simulation}

For the ionic kinetic energy regions (KER) considered in our experiment, there is only one electronic state of NO contributing. To find it, we perform a one-dimensional quantum simulation where the wave packet is propagated on the designated potential energy curves. Specifically, we follow the recipe of Ref. [6] and choose the $c^{3} \Pi, B^{1} \Pi$, $\mathrm{B}^{1} \Sigma^{+}$, and $4^{3} \Pi$ states, whose KER is within our present energy regime. Their corresponding potential energy curves are extracted from Ref. [31]. The cross section for photoionization starting from the initial ground $X^{2} \Pi$ state of the NO molecule is taken from Ref. [6]. The $X^{2} \Pi$ ground state is calculated using imaginary-time propagation, which, upon photoionization, is instantly populated onto the $\mathrm{c}^{3} \Pi, \mathrm{B}^{1} \Pi, \mathrm{B}^{1} \Sigma^{+}$, and $4^{3} \Pi$ states via the FranckCondon transition and evolves subsequently on the respective potential energy curve. The KER is obtained by Fourier transform of the wave packet when the internuclear distance is sufficiently large. The kinetic energy of the $\mathrm{N}^{+}$ fragment is then simply $\frac{16}{30} \mathrm{KER}$ according to the mass partitioning. The resulting correlated distribution of the photoelectron energy and the ionic energy is shown in 


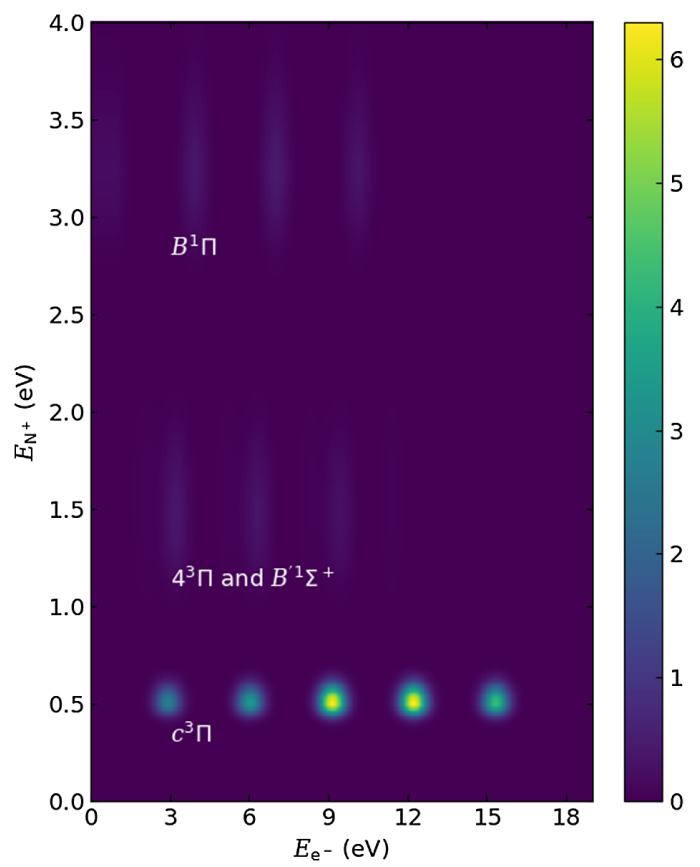

FIG. 12. Correlated distribution of the photoelectron energy and ionic energy.

Fig. 12, where the white text marks the corresponding pathway. It is clear from the figure that at an ionic energy of $0.4-0.5 \mathrm{eV}$, there is only one dominant pathway corresponding to dissociation on the $c^{3} \Pi$ state. Our theoretical simulation agrees well with our experimental results, as shown in Fig. 8, and the published results in Ref. [6].

Furthermore, we carry out a classical estimation of the rotation angle while the molecule breaks up. Following the supplemental materials of Ref. [26], the rotation angle can be written as

$\theta=\theta_{c}+\theta_{\infty}=c_{\omega}\left[\int_{t_{0}}^{t_{c}} \frac{1}{r(t)^{2}} d t+\int_{t_{c}}^{\infty} \frac{1}{r(t)^{2}} d t\right]$,

with the first term the rotation angle acquired in the first part of the dissociation process, where the potential energy curve is bumpy, and the second term corresponding to the flat part of the potential energy curve, where $r_{\mathrm{N}}=12$ is the border between these two parts. Here, $c_{\omega}$ is a constant for the NO molecule, which is estimated to be $6.816 \times$ $10^{-5}$ a.u. In this expression, the first term is calculated numerically using the cusp position of the wave packet on the $c^{3} \Pi$ state during the dissociation process as $r(t)$, and the second term is calculated analytically where $r(t)=r_{\mathrm{N}}+v_{c}\left(t-t_{c}\right)$, where $v_{c}=\sqrt{2 E_{\mathrm{N}}}$, with $E_{\mathrm{N}}$ the kinetic energy of $\mathrm{N}^{+}$, and $t_{c}$ is the time when the cusp of the wave packet reaches $r_{\mathrm{N}}$. With the present estimation, the rotation angle is calculated to be $0.95^{\circ}$. This small angle clarifies that the axial recoil approximation holds for this specific dissociation channel, as also shown in Ref. [6].

\section{APPENDIX C: NUCLEAR ROTATION EFFECT OF CO}

The $\mathrm{CO}$ and NO molecular ions differ in their potential energy curves. To verify the influence of the breakdown of the recoil axial approximation in the measurement of the molecular-frame angle-resolved photoionization time delays, we investigate the attosecond time-resolved photoionization dynamics of the $\mathrm{CO}$ molecule regarding its fast nuclear rotation dynamics in the dissociative ionization pathway of $\left(\mathrm{C}^{+}, \mathrm{O}\right)[75,76]$. Figure 13(a) shows the measured ion-momentum distribution, and Figs. 13(b) and 13(c) are the ion-momentum distributions belonging to the parallel and perpendicular pathways, which are separated via the ion recoil angle with respect to the polarization axis of the XUV-APT (along the $z$ axis). The coincidentally measured RABBITT spectra are plotted in Figs. 13(d)-13(f), respectively. Figures 14(a) and 14(b) show the ionic kinetic energy distributions of the parallel and perpendicular pathways. Figures 15(a)-15(c) show the measured joint kinetic energy spectra (JES) between electrons and ions under the photoionization from XUVAPT only, and Figs. 15(d)-15(f) show the results measured in the RABBITT situations.

As demonstrated in Refs. [75-77], in the photon energy range between $20 \mathrm{eV}$ and $36 \mathrm{eV}$, the dissociation pathway of $\left(\mathrm{C}^{+}, \mathrm{O}\right)$ is mostly generated following the photoionization of the $4 \sigma$ and $1 \pi$ orbitals via the electronic states of $B^{2} \Sigma^{+}(4 \sigma)^{-1}, 3^{2} \Sigma^{+}(4 \sigma)^{-1}, 4^{2} \Sigma^{+}(5 \sigma)^{-1}, D^{2} \Pi(1 \pi)^{-1}$, and $3^{2} \Pi(1 \pi)^{-1}$. The high vibrational states in the bound electronic state of $B^{2} \Sigma^{+}$could predissociate via the ${ }^{2} \Delta$ state, which generates the zero-kinetic-energy-realized ion fragments. The sharp peaks below $2 \mathrm{eV}$ correspond to the
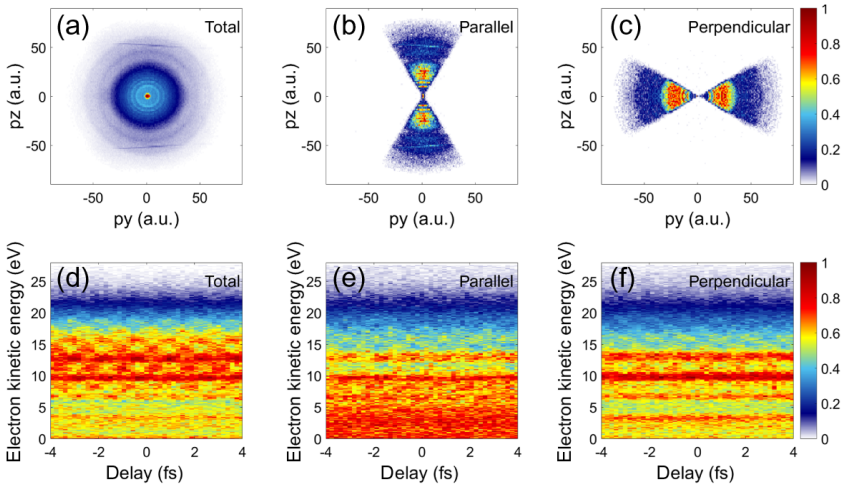

FIG. 13. Attosecond photoelectron spectra of the CO molecule. (a) Measured ion-momentum distribution of $\mathrm{C}^{+}$, where the $\mathrm{XUV}$ APT and NIR are both polarized along the $z$ axis. (b) Selected parallel-transition-associated ion-momentum distribution under the assumption of the axial recoil approximation with a cone angle of $\theta_{z}=30^{\circ}$. (c) Selected perpendicular-transition-associated ionmomentum distribution with a cone angle of $\theta_{y}=30^{\circ}$. (d)-(f) Corresponding measured RABBITT photoelectron spectra for each ion-momentum distribution shown in the upper row. 




FIG. 14. Ionic kinetic energy distribution. (a) Measured ionic kinetic energy distribution of the parallel transition pathway as selected in Fig. 13(b). (b) Same as (a) but for the perpendicular case.
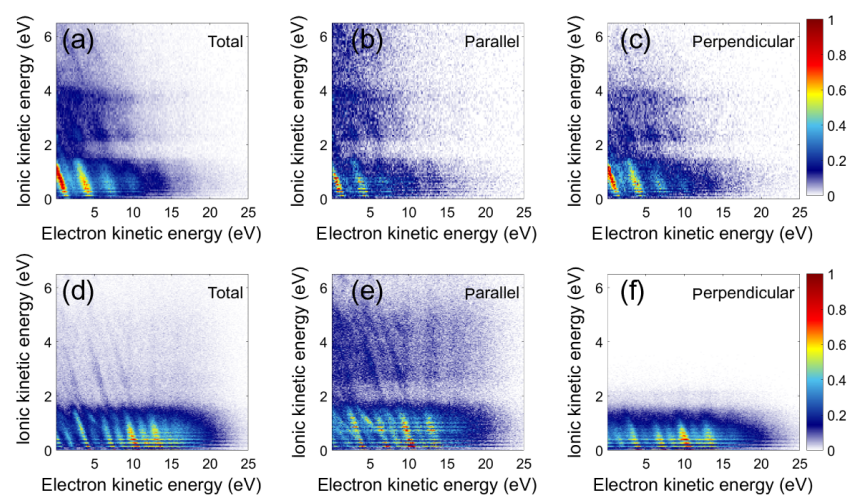

FIG. 15. Dissociative ionization pathway of the CO molecule. (a)-(c) Electron-ion joint energy spectrum (JES) of the dissociative ionization pathway of $\left(\mathrm{C}^{+}, \mathrm{O}\right)$ in the $\mathrm{CO}$ molecule under the XUV-APT only, where panel (a) shows the JES without any gating, panel (b) shows the JES gated by the parallel transition pathway, and panel (c) is the JES gated via the perpendicular transition pathway. (d,e) Same as the spectra in panels (a)-(c) but driven by the XUV-APT and NIR pulses together, where the pump-probe delay is integrated over the period of the NIR pulse.

homogeneously distributed ring structure in Fig. 13(a) originating from the vibrational-state-associated dissociation pathways, which is a strong hint of the breakdown of the axial recoil approximation.

Figures 16(a)-16(e) show the ion-momentum distributions with kinetic energy gating of $\left(0.0 \mathrm{eV}<E_{\mathrm{C}}<\right.$ $1.0 \mathrm{eV})$ in (a), $\left(1.0 \mathrm{eV}<E_{\mathrm{C}}<2.0 \mathrm{eV}\right)$ in (b), $(2.0 \mathrm{eV}<$ $\left.E_{\mathrm{C}}<3.0 \mathrm{eV}\right)$ in (c), $\left(3.0 \mathrm{eV}<E_{\mathrm{C}}<4.0 \mathrm{eV}\right)$ in (d), and $\left(4.0 \mathrm{eV}<E_{\mathrm{C}}<6.0 \mathrm{eV}\right)$ in (e), and their ion angular distributions are shown in Figs. 16(e)-16(h), respectively. A homogeneous ring structure shows up in the low kinetic energy range, below $2.0 \mathrm{eV}$, and the ion angular distribution shrinks to the laser polarization direction as the kinetic

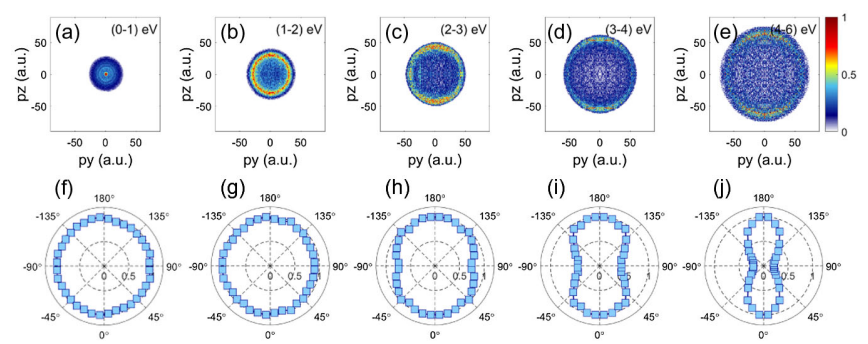

FIG. 16. $\mathrm{C}^{+}$ion-momentum distributions with kinetic energy gating. Panel (a) shows $0.0 \mathrm{~V}<E_{\mathrm{C}}<1.0 \mathrm{eV}$; panel (b), $1.0 \mathrm{eV}<$ $E_{\mathrm{C}}<2.0 \mathrm{eV}$; panel (c), $2.0 \mathrm{eV}<E_{\mathrm{C}}<3.0 \mathrm{eV}$; panel (d), $3.0 \mathrm{eV}<E_{\mathrm{C}}<4.0 \mathrm{eV}$; and panel (e), $4.0 \mathrm{eV}<E_{\mathrm{C}}<6.0 \mathrm{eV}$. (f)-(j) $C^{+}$ion angular distributions with the same kinetic energy selections as those for panels (a)-(e).

energy increases. The electronic-state-resolved ion angular momentum distributions in Fig. 17 also present a homogeneous distribution in the low kinetic energy range of the ions generated in the dissociation processes from the $3^{2} \Sigma^{+}$ and $D^{2} \Pi$ states in agreement with the previous study [75].
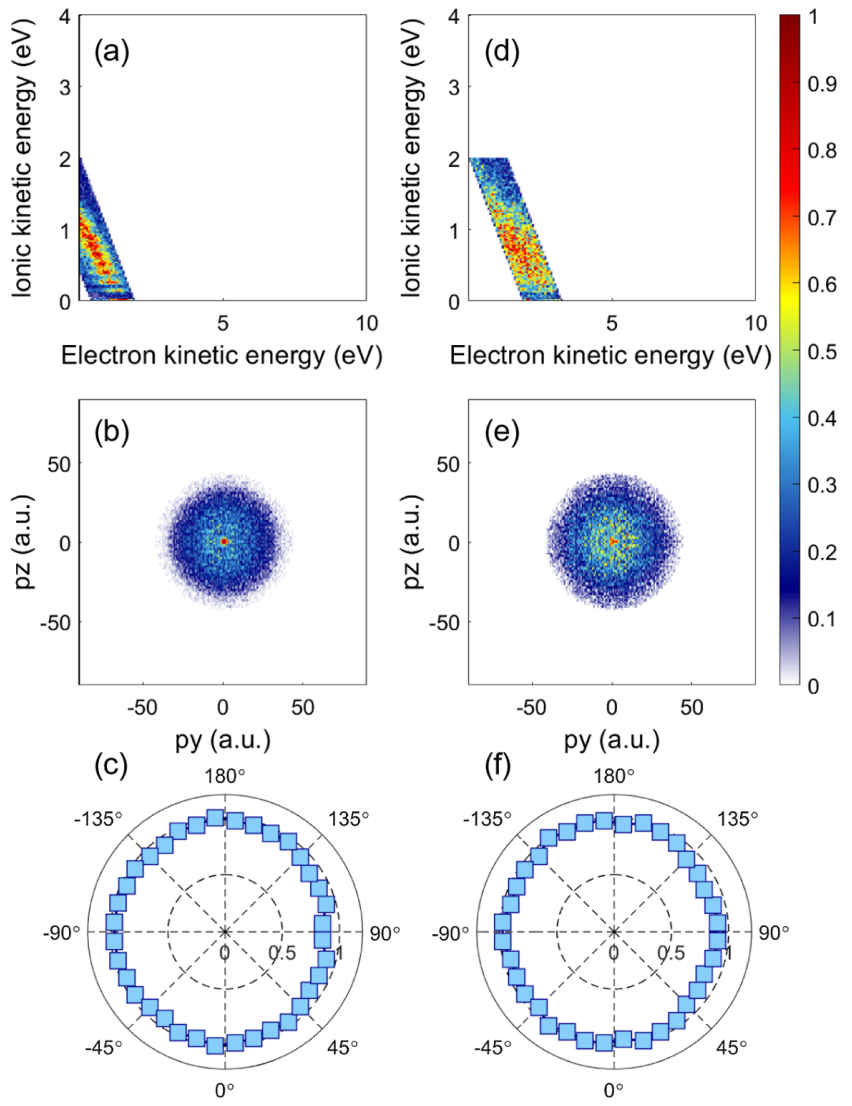

FIG. 17. Electronic-state-resolved angular distribution of the measured $\mathrm{C}^{+}$ions. (a)-(c) Ion-momentum distribution of $\mathrm{C}^{+}$ from the $D^{2} \Pi$ state. (a) Joint energy map between electrons and ions, (b) ion-momentum distribution, and (c) ion-fragment angular distribution. (d)-(f) Same as panels (a)-(c) but for the ion fragments from the $3^{2} \Sigma^{+}$electronic state. 
Employing the equation $I\left(\theta_{C}\right)=I_{0}\left\{1+\beta_{2} P_{2}\left[\cos \left(\theta_{C}\right)\right]\right\}$ to fit the angular distributions of the $\mathrm{C}^{+}$ion fragments in three kinetic energy ranges $0.0 \mathrm{eV}<E_{\mathrm{C}}<2.0 \mathrm{eV}$, $2.0 \mathrm{eV}<E_{\mathrm{C}}<3.5 \mathrm{eV}$, and $4.0 \mathrm{eV}<E_{\mathrm{C}}<6.0 \mathrm{eV}$, we obtain the asymmetric parameter $\beta_{2}$ for each harmonic. Figure 18 shows the resulting asymmetric parameter $\beta_{2}$ as a function of the XUV photon energy. We could separate two states $D^{2} \Pi$ and $3^{2} \Sigma^{+}$that appear in $0.0 \mathrm{eV}<E_{\mathrm{C}}<$ $2.0 \mathrm{eV}$. The isotropic ion distributions of $\mathrm{C}^{+}$ion fragments may be attributed either to a comparable weight between the parallel and the perpendicular components of the transition or to a significant predissociation time of the associated $\mathrm{CO}^{+}$ionic state [75]. Theoretical studies on these fragmentation channels suggest the predissociation timescale of about $0.3-0.5$ ps [76], illustrating the breakdown

of the axial recoil approximation for these dissociation channels.

For the molecular-frame photoemission study, a rule of thumb is suggested to exclude the low kinetic energy component of fragment ions that exhibits vibrational structure (see, e.g., Fig. 2 of Ref. [78]). Therefore, to investigate the molecular-frame angle-resolved photoionization time delays, one has to select ions in the high kinetic energy range, say, above about $3.0 \mathrm{eV}$ (see Figs. 14 and 16). The relative yield of the high kinetic energy range above about $3.0 \mathrm{eV}$ is, however, much lower than that in the low kinetic energy part below about $3.0 \mathrm{eV}$ (see Fig. 14). This gave us a big challenge for the molecular-frame photoionization time-delay measurements for $\mathrm{CO}$ based on the

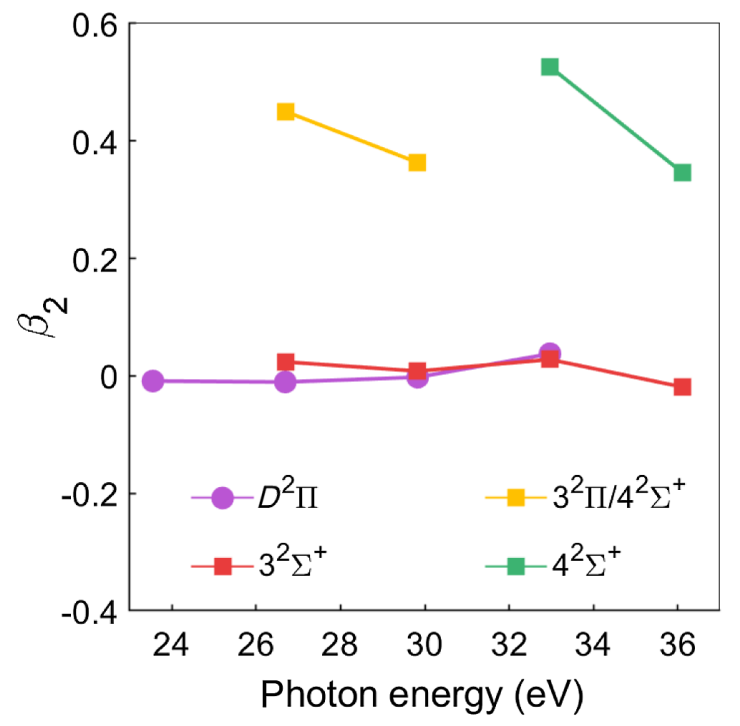

FIG. 18. Fitted asymmetric parameter $\beta_{2}$ of $\mathrm{C}^{+}$ion fragments dissociated from $D^{2} \Pi(1 \pi)^{-1}$ (purple line, $0.0 \mathrm{eV}<E_{\mathrm{C}}<2.0 \mathrm{eV}$ ), $3^{2} \Sigma^{+}(4 \sigma)^{-1} \quad$ (red line, $0.0 \mathrm{eV}<E_{\mathrm{C}}<2.0 \mathrm{eV}$ ), and $3^{2} \Pi(1 \pi)^{-1} / 4^{2} \Sigma^{+}(5 \sigma)^{-1}$ (yellow line, $2.0 \mathrm{eV}<E_{\mathrm{C}}<3.5 \mathrm{eV}$ ), where the $3^{2} \Pi$ and $4^{2} \Sigma^{+}$cannot be independently separated, and $4^{2} \Sigma^{+}(5 \sigma)^{-1}$ (green line, $4.0 \mathrm{eV}<E_{\mathrm{C}}<6.0 \mathrm{eV}$ ). electron-ion coincidence measurements. Furthermore, the predissociation, vibrational motion, and state coupling between electronic states that are populated in our photon energy range lead to a complicated electron-ion joint energy spectrum as shown in Fig. 15.

Figure 19(a) presents the measured time-delay difference between photoemission from the $\mathrm{C}$ end and the $\mathrm{O}$ end for the $\mathrm{CO}$ molecule through a standard parallel transition analysis as we performed for the NO molecule, and Fig. 19(b) shows the retrieved photoionization time delay under the perpendicular transition. The low kinetic energy range along the parallel transition pathway is mostly related to the $3^{2} \Sigma^{+}\left(4 \sigma^{-1}\right)$ state, whereas the perpendicular pathway is mostly related to the $D^{2} \Pi\left(1 \pi^{-1}\right)$ state. Our measured delays consist of the weak asymmetric signals as shown in Ref. [26] within the statistical measurement errors. As shown in Fig. 20, the photoionization from the $4 \sigma$ orbital exhibits a shape resonance at the photon energy around $36 \mathrm{eV}$. However, there is no significant asymmetric time delay in the parallel conditions as we observed in NO molecules. Depending on the specific electronic states
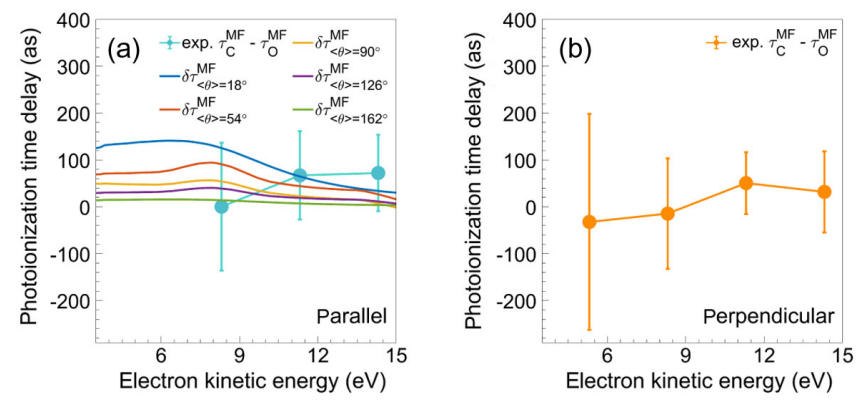

FIG. 19. Photoionization time delays of the CO molecule. (a) The retrieved asymmetric photoionization time delays under the parallel transition selection condition. (b) As same as (a) but for perpendicular transitions.
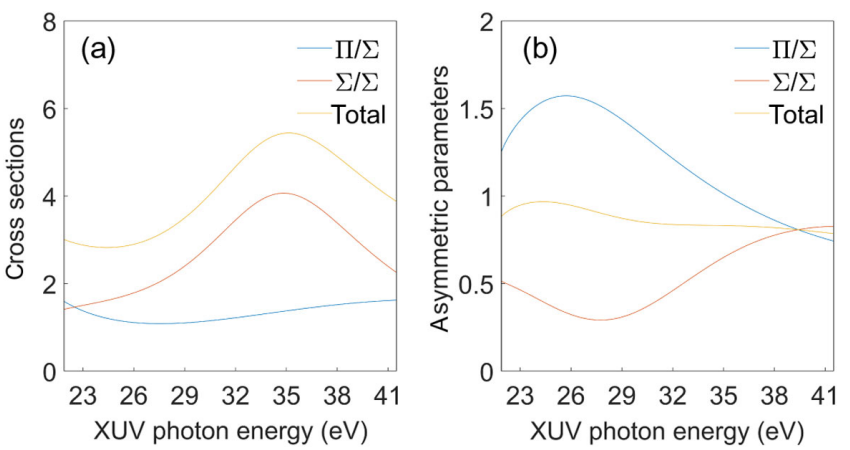

FIG. 20. Photoionization of the CO molecule. (a) The cross section distribution of the $4 \sigma$ electron photoionization in the CO molecule, where both the $\Sigma$ (red curve) and $\Pi$ (blue curve) continuum waves are identified. (b) The photoelectron asymmetric parameters distribution for the photoionization process as labeled in (a). 
excited by a laser pulse, the molecule may rotate, leading to the breakdown of the axial recoil approximation, which is exactly what we found in our $\mathrm{CO}$ measurements. To describe the nuclear rotation effects in the photoionization time delays, we perform a quantum scattering calculation in the $\mathrm{CO}$ molecule. The bound states of the $\mathrm{CO}$ molecule are calculated using an augmented-correlation-consistent polarized valence triplet zeta (aug-cc-pVTZ) basis set via the quantum chemistry package of MOLPRO [79-81]. The internuclear distance is fixed at $1.1379 \AA$. Both the initial and ionic states of $\mathrm{CO}$ molecules belong to $\Sigma$ symmetry during the photoionization of the $4 \sigma$ orbital. The theoretical result shows that the fast nuclear rotation smears out the asymmetric time delay along the nuclear axis. The present study on $\mathrm{CO}$ and $\mathrm{NO}$ illustrates that the molecular properties should be well identified to investigate the asymmetric photoionization dynamics in the molecular frame, and a proper sideband phase reconstruction routine, like the complex-valued principal component analysis illustrated in Appendix 1.B, is necessary for the multiorbital overlapped attosecond photoelectron spectroscopy.

\section{APPENDIX D: QUANTUM SCATTERING CALCULATIONS}

\section{Photoionization cross sections of the $4 \sigma$ electron in the NO molecule}

To describe the photoionization dynamics of an $N$-electron molecule, we employ a quantum scattering calculation based on the iterative Schwinger variational principle [60,82], where the electron wave function is decomposed through the one-center partial-wave expansion with maximal angular momentum up to 60 . The electronic structure of the molecule is treated at the Hartree-Fock (HF) level of the theory. To approach the exact Hartree-Fock limit as well as possible, we employ the largest basis set. In this approach, we perform a single-center partial-wave decomposition of the initial electronic state on a spherical grid, $\Psi_{i}=\sum_{\ell m} R_{n \ell}(r) Y_{\ell m}(\hat{r})$, which is constructed from an antisymmetrized product of the $n=N / 2$ occupied orbitals, as implemented in ePolyScat $[60,82]$. The finalstate wave function and the scattering potential are also expanded in partial waves. Here, the bound states of the NO molecule are calculated using an aug-cc-pVTZ basis set via the quantum chemistry package of MOLPRO [79-81]. The internuclear distance is fixed at 1.15077 angstrom. Because of the non-Abelian point group of the $C_{\infty v}$ symmetry of the NO molecule, a $C_{2 v}$ point group is used in the practical calculation, where both the initial and ionic states of the NO molecule belong to $B_{1}$ symmetry during the photoionization from the $4 \sigma$ orbital.

The photoionization dipole matrix elements in the length gauge for linearly polarized radiation are given by

$$
I_{i, f}=\left\langle\Psi_{f, \kappa}^{(-)}\left|\mathbf{r} \cdot \hat{\mathbf{E}}_{\mathrm{XUV}}\right| \Psi_{i}\right\rangle=\sqrt{\frac{4 \pi}{3 \kappa}} \sum_{\ell m v} I_{\ell m v} Y_{\ell m}(\hat{\kappa}) Y_{1 v}^{*}(\hat{\mathbf{n}}),
$$

where $\mathbf{r}$ is the position operator, $Y_{\ell m}$ is the spherical harmonics, $Y_{1, v=0, \pm 1}$ describes the orientation $\hat{\mathbf{E}}_{\mathrm{XUV}}$ of $\mathrm{XUV}$ polarization in the molecular frame, $\hat{\kappa}$ is the asymptotic momentum of the outgoing photoelectron wave packet, $\left|\Psi_{f, \kappa}^{(-)}\right\rangle$denotes the observed final-state wave function, and $I_{\ell m v}=\sqrt{(2 / \pi)}(-i)^{\ell}\left\langle\Psi_{f, \kappa \ell m}^{(-)}\left|\mathbf{r}_{v}\right| \Psi_{i}\right\rangle$ is the partialwave matrix element. The photoionization cross section integrated over target-orientation and photoelectronemission angles is given by

$$
\sigma=\frac{4 \pi^{2} E}{3 c} \sum\left|I_{i, f}\right|^{2}
$$

where $E$ is the photon energy. As shown in Fig. 21, the shape resonance occurs in the continuum electron wave with an $A_{1}$ symmetry, and the final scattering wave belongs to $B_{1}$ symmetry. Figure 22 shows the molecular-frame photoelectron angular distributions, which are calculated with the same electron kinetic energies from SB16 to SB22. A clear $\sigma$-type parallel transition along the $z$ axis (polarization direction) could be ascribed to the calculated MFPADs in Figs. 22(a)-22(d) with respect to the perpendicular transition in two other continuum waves of $B_{1}$ and $B_{2}$. In the following resonance search and molecular-frame photoionization time-delay calculations, we mainly focus on the continuum wave of $A_{1}$, which dominates the parallel photon transition in the vicinity of the shape resonance.

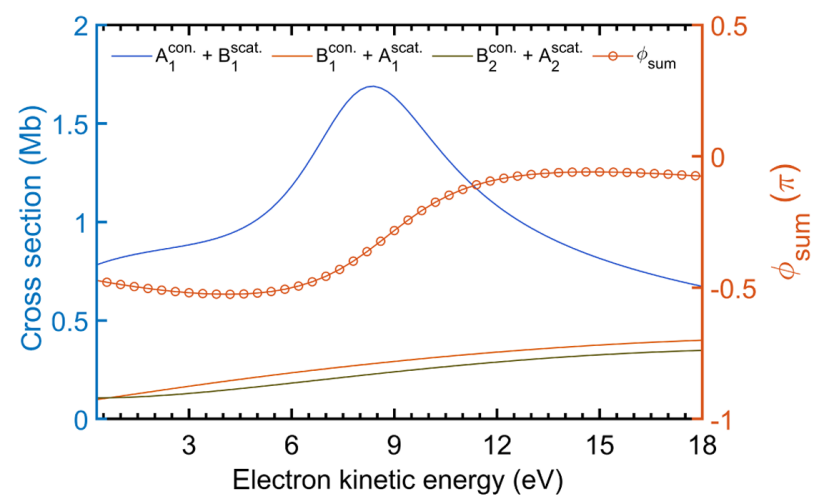

FIG. 21. Shape resonance of the NO molecule. The left axis shows the calculated photoionization cross section of the $4 \sigma$ electron of the NO molecule, including the continuum symmetries of $A_{1}, B_{1}$, and $B_{2}$. The enhanced cross-section variation around $9 \mathrm{eV}$ corresponds to the shape resonance position. The red dotted line shows the eigenphase sum calculated via the resonance search algorithm in ePolyScat $[60,82]$. 

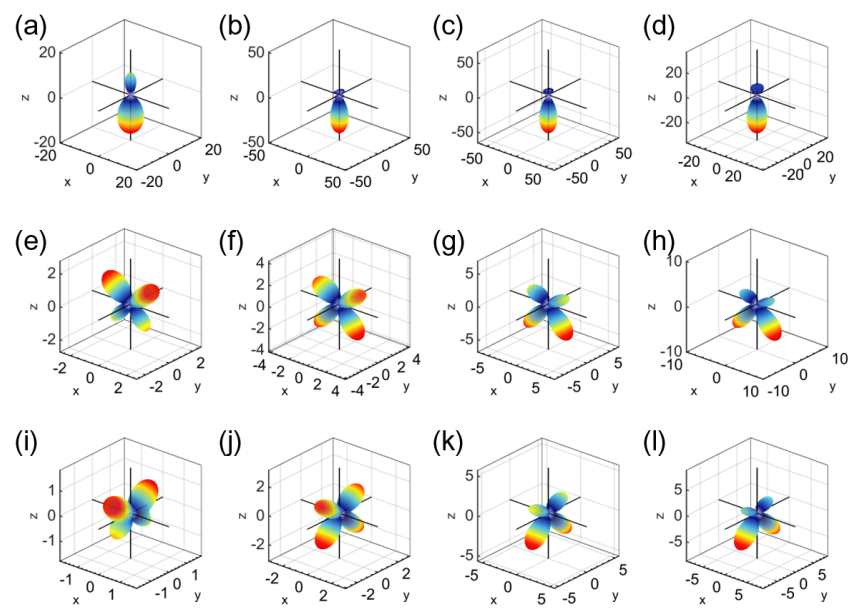

FIG. 22. Calculated molecular-frame photoelectron angular distributions. (a)-(d) MFPAD of the A1 continuum wave from SB16 to SB22, where the NO molecule is aligned along the $z$ axis with the $\mathrm{N}$ atom located on the positive side. (e)-(h) Same as in panels (a)-(d) but for the continuum wave of $B_{1}$. (i)-(l) Same as in panels (a)-(d) but for the continuum wave of $B_{2}$.

In general, a shape resonance is associated with a sudden jump in the eigenphase sum for the electron scattering dynamics. The eigenphase sum is approximately written as the sum of a constant background phase, $\delta_{0}$, and the resonance phase shifts as illustrated in Ref. [59]. The shape resonances are defined as poles of the scattering $\mathrm{S}$ matrix, where its energy dependence is analytically written into the complex plane. To figure out the shape resonance involved in the photoionization dynamics, we launch pole searches in the complex scattering-energy plane as developed in ePolyScat [59], which is shown as the red dotted line in Fig. 21. A sudden phase jump around $0.5 \pi$ is found across the peak of the shape resonance.

\section{Molecular-frame angle-resolved photoionization time-delay calculation}

The photoemission time-delay in the light-matter interaction is a fundamental question in attosecond science. As introduced by Wigner and Smith [24,25], the energy derivative of the scattering phase shift is associated with a time delay in scattering, called the EWS delay, $\tau_{\text {EWs }}$. In photoionization, this definition leads to a delay of the outgoing photoelectron wave packet. The Wigner phase shift is a reference physical quantity with respect to the motion of an ideal free electron wave packet. The cases of atoms and molecules have been treated in Refs. [71] and [57,58], respectively. The photoionization time delay is defined as the energy derivative of the complex photoionization amplitude $f(\varepsilon)$,

$$
\tau=\frac{d}{d \varepsilon} \operatorname{Arg}[f(\varepsilon)]=\operatorname{Im}\left\{\frac{1}{f(\varepsilon)} \frac{d f}{d \varepsilon}\right\} .
$$

In the picture of partial-wave decomposition, the outgoing wave packet is expressed as a coherent sum over partial waves, $\Psi=\sum_{l m} \psi_{l m}$, where each component is defined by the quantum numbers $(l, m)$, i.e., the electronic orbital angular momentum and its projection onto a given quantization axis, and each $(l, m)$ defines a partial-wave scattering channel.

As illustrated in Ref. [58], the two-photon ionization matrix element in the molecular frame is given by

$$
\begin{aligned}
& \mathbf{M}\left(\vec{k} ; \varepsilon_{i}+\Omega, \hat{\mathbf{R}}\right) \\
& =-i E_{\mathrm{XUV}} E_{\mathrm{IR}} \\
& \quad \times \sum_{p} \int d \varepsilon_{v} \frac{\left\langle\Psi_{f, \vec{k}}^{-}(\vec{r})\left|\vec{n}_{\mathrm{IR}} \cdot \vec{r}\right| \Psi_{v, p}\right\rangle\left\langle\Psi_{v, p}\left|\vec{n}_{\mathrm{XUV}} \cdot \vec{r}\right| \Psi_{i}\right\rangle}{\varepsilon_{i}+\Omega-\varepsilon_{v}}
\end{aligned}
$$

where $\Psi_{v, p}$ is the intermediate wave function. The angleand orientation-resolved intensity of a photoelectron sideband corresponding to energy $2 q \omega_{\text {IR }}$ created in an attosecond interferometry experiment is given by

$$
\begin{aligned}
\frac{d^{2} P_{2 q}}{d \hat{k} d \hat{R}_{\gamma}} \propto & \left|\mathbf{M}^{(2 q-1)}+\mathbf{M}^{(2 q+1)}\right|^{2} \\
= & \left|\mathbf{M}^{(2 q-1)}\right|^{2}+\left|\mathbf{M}^{(2 q+1)}\right|^{2} \\
& +2\left|\mathbf{M}^{(2 q-1)} \mathbf{M}^{(2 q+1)}\right| \cos \left[\operatorname{Arg}\left\{\mathbf{M}^{(2 q-1) *} \mathbf{M}^{(2 q+1)}\right\}\right] .
\end{aligned}
$$

The angle- and orientation-resolved time delay of the sideband photoelectron in the finite-difference approximation reads

$$
\tau\left(2 q, \hat{k}, \hat{R}_{\gamma}\right)=\frac{1}{2 \omega} \operatorname{Arg}\left(\mathbf{M}^{(2 q-1) *} \mathbf{M}^{(2 q+1)}\right) .
$$

The delay $\tau\left(2 q, \hat{k}, \hat{R}_{\gamma}\right)$ can be decomposed into a sum of two items of the continuum-continuum contribution $\tau_{\mathrm{cc}}(2 q)$ and the molecular scattering item $\tau_{\mathrm{mol}}\left(2 q, \hat{k}, \hat{R}_{\gamma}\right)$ :

$$
\tau\left(2 q, \hat{k}, \hat{R}_{\gamma}\right)=\tau_{\mathrm{cc}}(2 q)+\tau_{\mathrm{mol}}\left(2 q, \hat{k}, \hat{R}_{\gamma}\right) .
$$

The molecular-frame electron-ejection angle-resolved time delay is constructed under a certain molecular orientation direction in terms of

$$
\begin{aligned}
\tau_{\mathrm{mol}}^{\mathrm{MF}}(2 q, \hat{k})= & \frac{1}{2 \omega} \operatorname{Arg}\left[\sum_{\substack{L M \\
L^{\prime} M^{\prime}}} Y_{L^{\prime} M^{\prime}}^{*}(\hat{k}) Y_{L M}(\hat{k})\right. \\
& \left.\times \int d \hat{R}_{\gamma} A\left(\hat{R}_{\gamma}\right) b_{L^{\prime} M^{\prime} ;(2 q-1)}^{*}\left(\hat{R}_{\gamma}\right) b_{L M ;(2 q+1)}\left(\hat{R}_{\gamma}\right)\right] .
\end{aligned}
$$



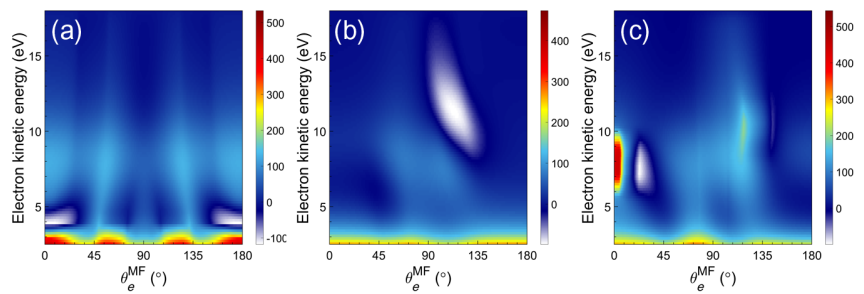

FIG. 23. Molecular-frame two-photon ionization time delays. (a) Angle-resolved photoionization time delay in the resonant channel with $L=2,4$. (b) Same as in (a) but for the nonresonant channel with $L=0,1,3$. (c) Same as in (a) but for the coherent superposition of all partial waves $(L=0,1,2,3,4)$.

Here, for the parallel transition along the molecular axis, we mainly consider the $4 \sigma-\sigma *$ transition in the continuum wave of $A_{1}$ symmetry. Figure 23 shows the partial-wave-resolved photoionization time delay related to the resonance channel, the nonresonance channel, and their coherent superposition. The resonance channel is defined with respect to the two-photon transition based on the partial-wave analysis of the cross section as shown in Fig. 1 in the main text, where the shape resonance occurs in the partial wave of $l=3$ during the parallel transition. However, the sidebands are generated via a two-photon process. The resonance channel is decomposed into $L=2$ and $L=4$ channels. Additionally, as shown in Fig. 24, the asymmetric photoionization time delay is also observed in the one-photon ionization time delays in the molecular frame. The laboratory frame angle-integrated two-photon ionization time delay [58] is the sum over all molecular orientations and the photoelectron emission angle in terms of

$$
\begin{aligned}
& \tau_{\mathrm{mol}}(2 q) \\
& \quad=\frac{1}{2 \omega} \operatorname{Arg}\left[\sum_{L M} \int d \hat{R}_{\gamma} b_{L M ;(2 q-1)}^{*}\left(\hat{R}_{\gamma}\right) b_{L M ;(2 q+1)}\left(\hat{R}_{\gamma}\right)\right] .
\end{aligned}
$$
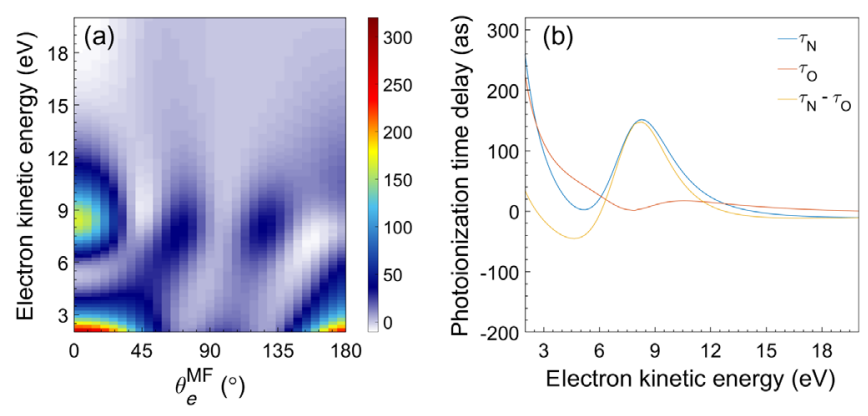

FIG. 24. One-photon ionization time delay. (a) Photoionization time delay as a function of photoelectron-ejection angle in the molecular frame in the vicinity of the molecular shape resonance. The molecule is oriented with the $\mathrm{N}$ atom along the positive polarization direction. (b) Site-resolved photoionization time delay along the $\mathrm{N}$ atom site (blue line) and the $\mathrm{O}$ atom site (red line) and their delay difference (yellow line).
To solve the complex issue of the continuum-continuum transition time delay in the vicinity of the shape resonance during the IR photon absorption, we consider the intramolecular photoionization time-delay difference between the $\mathrm{N}$ site and the $\mathrm{O}$ site, $\tau_{\mathrm{N}}-\tau_{\mathrm{O}}$, and the alternative case of the timedelay difference between the $\mathrm{N}$ site and the perpendicular direction, $\tau_{\mathrm{N}}-\tau_{\perp}$. As presented in the Fig. 2(a) of the main text, the delay difference between the $\mathrm{N}$ site and the $\mathrm{O}$ site shows a similar time-delay difference as a function of the photoelectron kinetic energy (or photon energy), which is only a small shift around 40 as. This indicates that the continuum-continuum time delays are not sensitive to the shape of the trapping potential since the photoelectron along the perpendicular transition pathway does not suffer from the trapping effect from the centrifugal potential barrier in the vicinity of the shape resonance.

\section{APPENDIX E: TWO-CENTER MULTIPLE SCATTERING ANALYSIS}

The photoionization time delay can be defined as the energy derivative of the phase of the transition dipole element

$$
\begin{aligned}
M_{\boldsymbol{k}} & =-i \int\left\langle\psi_{f}^{k} e^{-i \varepsilon_{k} t}|\boldsymbol{r} \cdot \boldsymbol{E}(t)| e^{i I_{p} t} \psi_{i}\right\rangle d t \\
& =-i\left\langle\psi_{f}^{k}|\boldsymbol{r} \cdot \boldsymbol{e}| \psi_{i}\right\rangle \int E(t) e^{i\left(\varepsilon_{\boldsymbol{k}}+I_{p}\right) t} d t,
\end{aligned}
$$

where $\psi_{i}$ and $\psi_{f}^{k}$ represent the initial and final wave functions, respectively, $\boldsymbol{E}(t)$ is the electric field of the laser pulse with $\boldsymbol{e}$ its polarization direction, $I_{p}$ is the ionization energy of the initial state, and $\varepsilon_{\boldsymbol{k}}=\boldsymbol{k}^{2} / 2$ is the energy of the electron with momentum $\boldsymbol{k}$. For a transform-limited chirp-free pulse, the phase from the Fourier transform of the laser pulse is trivial and can be ignored, and the photoionization time delay is simply

$$
\tau=\frac{d}{d \varepsilon} \arg \left\{\left\langle\psi_{i}|\boldsymbol{r} \cdot \boldsymbol{e}| \psi_{f}^{k}\right\rangle\right\} .
$$

Following Refs. [83,84], we treat the final state as a multiple scattering expansion of the plane wave impinging on the $\mathrm{N}$ and $\mathrm{O}$ atoms of the $\mathrm{NO}$ molecule. For the present purpose of describing the asymmetric emission time delay from different atoms, we expand to the order of third scattering. Thereby, the final state can be written as a sum of

$$
\psi_{f, 0}^{k}(\boldsymbol{r})=e^{i \boldsymbol{k} \cdot \boldsymbol{r}},
$$

$$
\begin{aligned}
& \psi_{f, \mathrm{~N} 1}^{\boldsymbol{k}}(\boldsymbol{r})=\frac{e^{i k\left|\boldsymbol{r}-\boldsymbol{R}_{\mathrm{N}}\right|}}{\left|\boldsymbol{r}-\boldsymbol{R}_{\mathrm{N}}\right|} f_{\mathrm{N}}\left[\theta\left(\boldsymbol{r}-\boldsymbol{R}_{\mathrm{N}}, \boldsymbol{k}\right)\right] e^{i \boldsymbol{k} \cdot \boldsymbol{R}_{\mathrm{N}}}, \\
& \psi_{f, \mathrm{O} 1}^{\boldsymbol{k}}(\boldsymbol{r})=\frac{e^{i k\left|\boldsymbol{r}-\boldsymbol{R}_{\mathrm{O}}\right|}}{\left|\boldsymbol{r}-\boldsymbol{R}_{\mathrm{O}}\right|} f_{\mathrm{O}}\left[\theta\left(\boldsymbol{r}-\boldsymbol{R}_{\mathrm{O}}, \boldsymbol{k}\right)\right] e^{i \boldsymbol{k} \cdot \boldsymbol{R}_{\mathrm{O}}},
\end{aligned}
$$




$$
\psi_{f, \mathrm{~N} 2}^{k}(\boldsymbol{r})=\frac{e^{i k\left|\boldsymbol{r}-\boldsymbol{R}_{\mathrm{N}}\right|}}{\left|\boldsymbol{r}-\boldsymbol{R}_{\mathrm{N}}\right|} f_{\mathrm{N}}\left[\theta\left(\boldsymbol{r}-\boldsymbol{R}_{\mathrm{N}}, \boldsymbol{k}\right)\right] \frac{e^{i k R}}{R} f_{\mathrm{O}}[\theta(\boldsymbol{R}, \boldsymbol{k})] e^{i \boldsymbol{k} \cdot \boldsymbol{R}_{\mathrm{O}}},
$$

$$
\psi_{f, \mathrm{O} 2}^{\boldsymbol{k}}(\boldsymbol{r})=\frac{e^{i k\left|\boldsymbol{r}-\boldsymbol{R}_{\mathrm{O}}\right|}}{\left|\boldsymbol{r}-\boldsymbol{R}_{\mathrm{O}}\right|} f_{\mathrm{O}}\left[\theta\left(\boldsymbol{r}-\boldsymbol{R}_{\mathrm{O}}, \boldsymbol{k}\right)\right] \frac{e^{i k R}}{R} f_{\mathrm{N}}[\theta(-\boldsymbol{R}, \boldsymbol{k})] e^{i \boldsymbol{k} \cdot \boldsymbol{R}_{\mathrm{N}}},
$$

$$
\begin{aligned}
\psi_{f, \mathrm{~N} 3}^{\boldsymbol{k}}(\boldsymbol{r})= & \frac{e^{i k\left|\boldsymbol{r}-\boldsymbol{R}_{\mathrm{N}}\right|}}{\left|\boldsymbol{r}-\boldsymbol{R}_{\mathrm{N}}\right|} f_{\mathrm{N}}\left[\theta\left(\boldsymbol{r}-\boldsymbol{R}_{\mathrm{N}}, \boldsymbol{k}\right)\right] \frac{e^{i k R}}{R} f_{\mathrm{O}}[\theta(\boldsymbol{R}, \boldsymbol{k})] \\
& \times \frac{e^{i k R}}{R} f_{\mathrm{N}}[\theta(-\boldsymbol{R}, \boldsymbol{k})] e^{i \boldsymbol{k} \cdot \boldsymbol{R}_{\mathrm{N}}}, \\
\psi_{f, \mathrm{O} 3}^{\boldsymbol{k}}(\boldsymbol{r})= & \frac{e^{i k\left|\boldsymbol{r}-\boldsymbol{R}_{\mathrm{O}}\right|}}{\left|\boldsymbol{r}-\boldsymbol{R}_{\mathrm{O}}\right|} f_{\mathrm{O}}\left[\theta\left(\boldsymbol{r}-\boldsymbol{R}_{\mathrm{O}}, \boldsymbol{k}\right)\right] \frac{e^{i k R}}{R} f_{\mathrm{N}}[\theta(-\boldsymbol{R}, \boldsymbol{k})] \\
& \times \frac{e^{i k R}}{R} f_{\mathrm{O}}[\theta(\boldsymbol{R}, \boldsymbol{k})] e^{i \boldsymbol{k} \cdot \boldsymbol{R}_{\mathrm{O}}},
\end{aligned}
$$

where $\psi_{f, 0}^{k}(\boldsymbol{r})$ is the plane wave with momentum $\boldsymbol{k}, \psi_{f, \mathrm{~N} 1}^{\boldsymbol{k}}(\boldsymbol{r})$ is its first scattering from the $\mathrm{N}$ atom, and $\psi_{f, \mathrm{O} 1}^{k}(\boldsymbol{r})$ is the first scattering from the $\mathrm{O}$ atom, where $\boldsymbol{R}_{\mathrm{N}}$ and $\boldsymbol{R}_{\mathrm{O}}$ are the location vectors of the $\mathrm{N}$ and $\mathrm{O}$ atoms, $f_{\mathrm{N}}$ and $f_{\mathrm{O}}$ are the respective scattering amplitudes, which are functions of the scattering angle $\theta$ defined between $\boldsymbol{r}-\boldsymbol{R}_{\mathrm{N} / \mathrm{O}}$ and $\boldsymbol{k}$, and $\boldsymbol{R}=\boldsymbol{R}_{\mathrm{N}}-\boldsymbol{R}_{\mathrm{O}}$, with $R$ its magnitude.

When the molecular axis aligns with the laser polarization direction, the scattering amplitudes from the $\mathrm{N}$ and $\mathrm{O}$ atoms can be expanded in partial waves as

$$
\begin{aligned}
f_{\mathrm{N} / \mathrm{O}}(\theta) & =\sum_{l}(2 l+1) \frac{e^{2 i \delta_{l}^{\mathrm{N} / \mathrm{O}}(k)}-1}{2 i k} P_{l}(\cos \theta) \\
& =\sum_{l}(2 l+1) \frac{\sin \delta_{l}^{\mathrm{N} / \mathrm{O}}}{k} e^{i \delta_{l}^{\mathrm{N} / \mathrm{O}}(k)} P_{l}(\cos \theta),
\end{aligned}
$$

where $P_{l}$ is the Legendre function, and $\delta_{l}^{\mathrm{N} / \mathrm{O}}(k)$ is the scattering phase shift for the $\mathrm{N}$ and $\mathrm{O}$ atoms for partial wave $l$. For a diatomic molecule, we need to distinguish the partial wave for the molecular final state and the partial wave by individual atoms in the context of two-center expansion. It is well known that the shape resonance is most prominent for the $l=3$ partial scattering channel, which, for a diatomic molecule, is built up by the $l=1$ partial-wave channel of individual atoms [59]. Thus, we sum the partial scattering amplitude from $l=0$ up to a maximum of $l=1$ for each atom. From Eq. (E10), it is clear that a single partial-wave channel $l$ from a single atom has an isotropic phase $\delta_{l}$, producing an isotropic atomic time delay. When different $l$ channels, including $l=0,1$ for individual atoms and that from different atoms, are coherently summed up, the angular dependence of time delay emerges.

To obtain accurate phase shifts for different $l$ partialwave channels at the energy regime of interest of the present work, we integrate the radial Schrödinger equation

$$
\frac{d^{2}}{d r^{2}} \chi(r)=2\left[V(r)+\frac{l(l+1)}{2 r^{2}}-\varepsilon\right] \chi(r)
$$

using the Numerov method [85], and the phase shift $\delta_{l}(k)$ of the radial wave function $\chi(r)$ is obtained by comparing to the plane wave. Since the $\mathrm{N}$ and $\mathrm{O}$ atoms have different atomic potentials $V(r)$, their corresponding phase shifts differ, resulting in the observed asymmetric time delay when the electron is emitted from different sides. Specifically, we have used the following atomic potentials:

$$
\begin{aligned}
& V_{\mathrm{N}}(r)=-\left(0.3615+6.6385 e^{-1.75 r}\right) / r, \\
& V_{\mathrm{O}}(r)=-\left(0.6385+7.3615 e^{-2.00 r}\right) / r,
\end{aligned}
$$

which are obtained by fitting to the electron-molecule interaction potential of $\mathrm{NO}$ calculated using the adiabatic local model potential [59] using the same target wave function as was used in the full quantum scattering calculations. The scattering phase shifts $\delta_{l}$, the associated time delays $\tau$, and magnitudes of the scattering amplitude $f_{l}$ for the $\mathrm{N}$ and $\mathrm{O}$ atoms are shown in Fig. 25. Here, the partial-wave scattering amplitude $f_{l}$ follows the convention that $f_{l}=(2 l+1)\left(e^{2 i \delta_{l}}-1\right) / 2 i k$. It is obvious that the different phase shifts for the $\mathrm{N}$ and $\mathrm{O}$ atoms lead to very
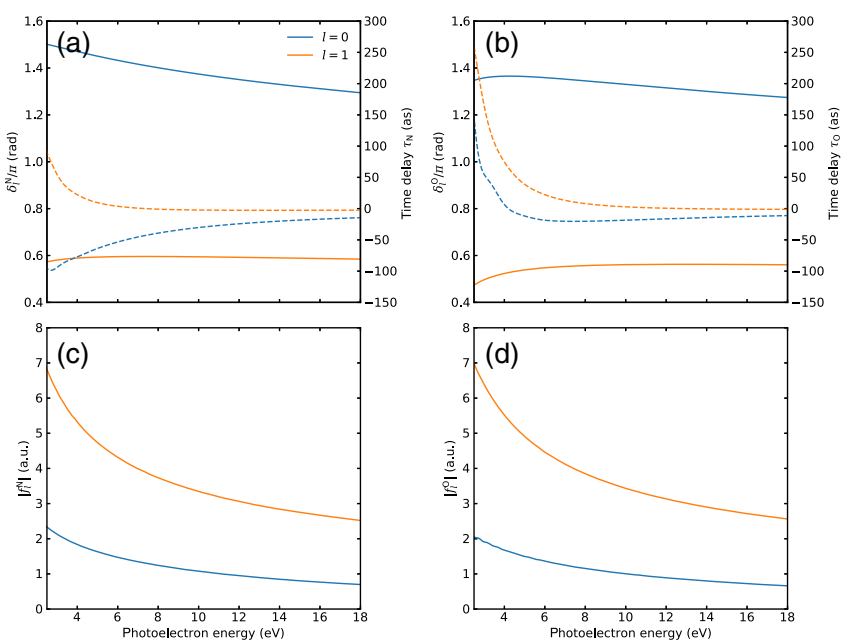

FIG. 25. Partial-wave-resolved photoelectron scattering dynamics. Scattering phase shifts (upper row, left ordinate, solid lines) and the associated time delays (upper row, right ordinate, dashed lines) and magnitudes of the scattering amplitude (lower row) for different partial-wave channels of the individual $\mathrm{N}$ (left column) and $\mathrm{O}$ (right column) atoms. 
different emission time delays and scattering amplitudes for individual atoms.

The initial state of the $4 \sigma$ orbital used in the two-center model is obtained using a very simple STO-3G basis set in a self-consistent field calculation on the ground state of NO. The localized initial wave functions for $\mathrm{N} / \mathrm{O}$ are denoted as $\psi_{i, \mathrm{~N} / \mathrm{O}}$, so the full initial state is written as $\psi_{i}=\psi_{i, \mathrm{~N}}+\psi_{i, \mathrm{O}}$, where the localized wave functions are defined in terms of the expansion of the STO-3G functions on each center.

In the spirit of the Muffin-Tin model, the transition dipole element should be an overlap between the initial state at $\mathrm{N}$ and the scattering wave from $\mathrm{O}$ and vice versa; thus,

$$
\begin{aligned}
M_{\boldsymbol{k}}= & \left\langle\psi_{i, \mathrm{~N}}(\boldsymbol{r})|\boldsymbol{e} \cdot \boldsymbol{r}| \psi_{f, 0}+\psi_{f, \mathrm{O} 1}^{\boldsymbol{k}}(\boldsymbol{r})+\psi_{f, \mathrm{O} 2}^{\boldsymbol{k}}(\boldsymbol{r})+\psi_{f, \mathrm{O} 3}^{\boldsymbol{k}}(\boldsymbol{r})\right\rangle \\
& +\left\langle\psi_{i, \mathrm{O}}(\boldsymbol{r})|\boldsymbol{e} \cdot \boldsymbol{r}| \psi_{f, 0}+\psi_{f, \mathrm{~N} 1}^{\boldsymbol{k}}(\boldsymbol{r})+\psi_{f, \mathrm{~N} 2}^{\boldsymbol{k}}(\boldsymbol{r})+\psi_{f, \mathrm{~N} 3}^{\boldsymbol{k}}(\boldsymbol{r})\right\rangle .
\end{aligned}
$$

After the phase shifts for the $\mathrm{N}$ and $\mathrm{O}$ atoms are calculated, they are plugged into Eq. (E10), which is in turn plugged into Eq. (E14) to obtain the transition dipole element $M_{k}$. In the end, the photoionization time delay is obtained as $\tau=d \arg \left\{M_{k}\right\} / d \varepsilon$.

The present two-center multiple scattering analysis allows us to gain further insights into the shape resonance and the associated asymmetric time delay. In order to pin down the origin of the asymmetry in the photoemission delay, we selectively make the initial and/or the final state symmetric or asymmetric. Here, we may manually set the scattering phase shift of $\mathrm{O}$ to be the same as $\mathrm{N}$, such that the final scattering state is symmetric. We may also artificially set the initial state to have the same form; for example, we may set $\psi_{i, \mathrm{~N}}=\exp \left\{-r_{\mathrm{N}}\right\}$ and $\psi_{i, \mathrm{O}}=-\exp \left\{-r_{\mathrm{O}}\right\}$, where $r_{\mathrm{N} / \mathrm{O}}$ is the distance to the N/O atom. Setting both the initial and final states to be symmetric would mimic the shape resonance observed in $\mathrm{N}_{2}$. As found in the main text, the shape resonance is still present, which leads to the bump in the time delay at the corresponding energy region while the asymmetry in the emission delay vanishes. Studying different combinations of symmetric and/or asymmetric initial/final states, we find that the asymmetry in both the initial and final states plays a role in the asymmetric time delay, with electrons emitting in different directions. While the asymmetric time delay is largely due to the asymmetric final scattering state, the location of the shape resonance where the delay asymmetry is maximized is sensitive to the asymmetry of the initial state.

In the present two-center picture, it is the heteronuclear nature of the molecule that leads to the asymmetric delay. By "heteronuclear," we are referring to the different potentials around the two atoms. The heteronuclear nature leads to both an asymmetry in the initial state and an asymmetry in the final state. From a symmetry point of view, both of these factors must contribute to the observed asymmetric delay. As to which factor contributes more and/or leads to what changes, it depends on the relative importance of these two factors, which certainly differ for different molecules. Thus, it is not surprising that the asymmetric initial state of $\mathrm{CO}$ mostly leads to the asymmetric delay in $\mathrm{CO}$ [26], while it is the asymmetric final state of NO that mostly results in the asymmetric delay in NO.

The one-center and two-center pictures are the two complementary ways to explain the observed asymmetric angle-dependent photoemission time delay. An analogue, though maybe not very proper, would be multiphoton ionization and tunneling ionization for an atom. In multiphoton ionization, the coherent summation of different above-threshold ionization peaks gives rise to asymmetric photoelectron momentum distribution, and in tunneling ionization, it is the asymmetry in the laser field that leads to the asymmetric momentum distribution. We know that multiphoton ionization is the frequency-domain interpretation of strong-field ionization, and tunneling ionization is the time-domain interpretation. For the present work, the one-center partial-wave analysis is a frequency-domain interpretation, and the two-center asymmetry analysis is a time-domain (or rather, spatial-domain) interpretation of the asymmetric angle-dependent time delay. Both interpretations are valid, and which interpretation to use is only a matter of taste and convenience.

[1] J. L. Dehmer, Evidence of Effective Potential Barriers in the X-Ray Absorption Spectra of Molecules, J. Chem. Phys. 56, 4496 (1972).

[2] G. J. Schulz, Resonances in Electron Impact on Atoms, Rev. Mod. Phys. 45, 378 (1973).

[3] J. M. Combes, P. Duclos, M. Klein, and R. Seiler, The Shape Resonance, Commun. Math. Phys. 110, 215 (1987).

[4] E. Shigemasa, J. Adachi, K. Soejima, N. Watanabe, A. Yagishita, and N. A. Cherepkov, Direct Determination of Partial Wave Contributions in the $\sigma *$ Shape Resonance of CO Molecules, Phys. Rev. Lett. 80, 1622 (1998).

[5] M. N. Piancastelli, The Never Ending Story of Shape Resonances, J. Electron Spectrosc. Relat. Phenom. 100, 167 (1999).

[6] K. Veyrinas, N. Saquet, S. Marggi Poullain, M. Lebech, J. C. Houver, R. R. Lucchese, and D. Dowek, Dissociative Photoionization of NO across a Shape Resonance in the XUV Range Using Circularly Polarized Synchrotron Radiation, J. Chem. Phys. 151, 174305 (2019).

[7] J. H. Fock and E. E. Koch, Shape Resonances and Partial Photoemission Cross Sections of Solid $\mathrm{SF}_{6}$ and $\mathrm{CCl}_{4}$, Chem. Phys. 96, 125 (1985).

[8] Y. Pertot, C. Schmidt, M. Matthews, A. Chauvet, M. Huppert, V. Svoboda, A. Von Conta, A. Tehlar, D. Baykusheva, J. P. Wolf, and H.J. Wörner, Time-Resolved X-Ray Absorption Spectroscopy with a Water Window High-Harmonic Source, Science 355, 264 (2017). 
[9] E. Plésiat, S. E. Canton, J. D. Bozek, P. Decleva, and F. Martín, Resonant Photoelectron Confinement in the $\mathrm{SF}_{6}$ Molecule, J. Phys. Chem. A 123, 1062 (2019).

[10] R. E. Palmer and P. J. Rous, Resonances in Electron Scattering by Molecules on Surfaces, Rev. Mod. Phys. 64, 383 (1992).

[11] A. Ferré, A. E. Boguslavskiy, M. Dagan, V. Blanchet, B. D. Bruner, F. Burgy, A. Camper, D. Descamps, B. Fabre, N. Fedorov, J. Gaudin, G. Geoffroy, J. Mikosch, S. Patchkovskii, S. Petit, T. Ruchon, H. Soifer, D. Staedter, I. Wilkinson, A. Stolow, N. Dudovich, and Y. Mairesse, Multi-Channel Electronic and Vibrational Dynamics in Polyatomic Resonant High-Order Harmonic Generation, Nat. Commun. 6, 5952 (2015).

[12] K. A. Larsen, C. S. Trevisan, R. R. Lucchese, S. Heck, W. Iskandar, E. Champenois, A. Gatton, R. Moshammer, R. Strom, T. Severt, B. Jochim, D. Reedy, M. Weller, A. L. Landers, J. B. Williams, I. Ben-Itzhak, R. Dörner, D. Slaughter, C. W. McCurdy, T. Weber, and T. N. Rescigno, Resonance Signatures in the Body-Frame Valence Photoionization of $\mathrm{CF}_{4}$, Phys. Chem. Chem. Phys. 20, 21075 (2018).

[13] J. Rist, K. Klyssek, N. M. Novikovskiy, M. Kircher, I. VelaPérez, D. Trabert, S. Grundmann, D. Tsitsonis, J. Siebert, A. Geyer, N. Melzer, C. Schwarz, N. Anders, L. Kaiser, K. Fehre, A. Hartung, S. Eckart, L. P. H. Schmidt, M. S. Schöffler, V. T. Davis, J. B. Williams, F. Trinter, R. Dörner, P. V. Demekhin, and T. Jahnke, Measuring the Photoelectron Emission Delay in the Molecular Frame, arXiv:2107.05994.

[14] F. Holzmeier, J. Joseph, J. C. Houver, M. Lebech, D. Dowek, and R. R. Lucchese, What Causes the Angular Dependence of Pho-toionization Time Delays in a Shape Resonance?, arXiv:2107.09915.

[15] S. Haessler, B. Fabre, J. Higuet, J. Caillat, T. Ruchon, P. Breger, B. Carré, E. Constant, A. Maquet, E. Mével, P. Salières, R. Taïeb, and Y. Mairesse, Phase-Resolved Attosecond Near-Threshold Photoionization of Molecular Nitrogen, Phys. Rev. A 80, 011404(R) (2009).

[16] P. M. Kraus, D. Baykusheva, and H. J. Wörner, TwoPulse Field-Free Orientation Reveals Anisotropy of Molecular Shape Resonance, Phys. Rev. Lett. 113, 023001 (2014).

[17] M. Huppert, I. Jordan, D. Baykusheva, A. von Conta, and H. J. Wörner, Attosecond Delays in Molecular Photoionization, Phys. Rev. Lett. 117, 093001 (2016).

[18] S. Nandi, E. Plésiat, S. Zhong, A. Palacios, D. Busto, M. Isinger, L. Neoričić, C. L. Arnold, R. J. Squibb, R. Feifel, P. Decleva, A. L'Huillier, F. Martín, and M. Gisselbrecht, Attosecond Timing of Electron Emission from a Molecular Shape Resonance, Sci. Adv. 6, eaba7762 (2020).

[19] M. Hentschel, R. Kienberger, C. Spielmann, G. A. Reider, N. Milosevic, T. Brabec, P. Corkum, U. Heinzmann, M. Drescher, and F. Krausz, Attosecond Metrology, Nature (London) 414, 509 (2001).

[20] J. Itatani, F. Quéré, G. L. Yudin, M. Y. Ivanov, F. Krausz, and P. B. Corkum, Attosecond Streak Camera, Phys. Rev. Lett. 88, 173903 (2002).

[21] R. Pazourek, S. Nagele, and J. Burgdörfer, Attosecond Chronoscopy of Photoemission, Rev. Mod. Phys. 87, 765 (2015).
[22] P. M. Paul, E. S. Toma, P. Breger, G. Mullot, F. Augé, P. Balcou, H. G. Muller, and P. Agostini, Observation of a Train of Attosecond Pulses from High Harmonic Generation, Science 292, 1689 (2001).

[23] S. Nagele, R. Pazourek, J. Feist, and J. Burgdörfer, Time Shifts in Photoemission from a Fully Correlated TwoElectron Model System, Phys. Rev. A 85, 033401 (2012).

[24] E. P. Wigner, Lower Limit for the Energy Derivative of the Scattering Phase Shift, Phys. Rev. 98, 145 (1955).

[25] F. T. Smith, Lifetime Matrix in Collision Theory, Phys. Rev. 118, 349 (1960).

[26] J. Vos, L. Cattaneo, S. Patchkovskii, T. Zimmermann, C. Cirelli, M. Lucchini, A. Kheifets, A. S. Landsman, and U. Keller, Orientation-Dependent Stereo Wigner Time Delay and Electron Localization in a Small Molecule, Science 360, 1326 (2018).

[27] K. L. Reid, Photoelectron Angular Distributions: Developments in Applications to Isolated Molecular Systems, Mol. Phys. 110, 131 (2012).

[28] M. Kunitski, N. Eicke, P. Huber, J. Köhler, S. Zeller, J. Voigtsberger, N. Schlott, K. Henrichs, H. Sann, F. Trinter, L. P. H. Schmidt, A. Kalinin, M. S. Schöffler, T. Jahnke, M. Lein, and R. Dörner, Double-Slit Photoelectron Interference in Strong-Field Ionization of the Neon Dimer, Nat. Commun. 10, 1 (2019).

[29] R. Dörner, V. Mergel, O. Jagutzki, L. Spielberger, J. Ullrich, R. Moshammer, and H. Schmidt-Böcking, Cold Target Recoil Ion Momentum Spectroscopy: A “Momentum Microscope” to View Atomic Collision Dynamics, Phys. Rep. 330, 95 (2000).

[30] X. Gong, C. Lin, F. He, Q. Song, K. Lin, Q. Ji, W. Zhang, J. Ma, P. Lu, Y. Liu, H. Zeng, W. Yang, and J. Wu, EnergyResolved Ultrashort Delays of Photoelectron Emission Clocked by Orthogonal Two-Color Laser Fields, Phys. Rev. Lett. 118, 143203 (2017).

[31] Y. Hikosaka, T. Aoto, K. Ito, Y. Terasaka, R. Hirayama, and E. Miyoshi, Threshold Photoelectron Spectroscopy on Inner-Valence Ionic States of NO, J. Chem. Phys. 128, 044320 (2008).

[32] H. G. Muller, Reconstruction of Attosecond Harmonic Beating by Interference of Two-Photon Transitions, Appl. Phys. B 74, s17 (2002).

[33] M. Isinger, R. J. Squibb, D. Busto, S. Zhong, A. Harth, D. Kroon, S. Nandi, C. L. Arnold, M. Miranda, J. M. Dahlström, E. Lindroth, R. Feifel, M. Gisselbrecht, and A. L'Huillier, Photoionization in the Time and Frequency Domain, Science 358, 893 (2017).

[34] M. Drescher, M. Hentschel, R. Kienberger, M. Uiberacker, V. Yakovlev, A. Scrinzi, T. Westerwalbesloh, U. Kleineberg, U. Heinzmann, and F. Krausz, Time-Resolved Atomic InnerShell Spectroscopy, Nature (London) 419, 803 (2002).

[35] A. S. Kheifets and I. A. Ivanov, Delay in Atomic Photoionization, Phys. Rev. Lett. 105, 233002 (2010).

[36] M. Swoboda, T. Fordell, K. Klünder, J. M. Dahlström, M. Miranda, C. Buth, K. J. Schafer, J. Mauritsson, A. L'Huillier, and M. Gisselbrecht, Phase Measurement of Resonant Two-Photon Ionization in Helium, Phys. Rev. Lett. 104, 103003 (2010).

[37] A. Kaldun, A. Blättermann, V. Stooß, S. Donsa, H. Wei, R. Pazourek, S. Nagele, C. Ott, C. D. Lin, J. Burgdörfer, and 
T. Pfeifer, Observing the Ultrafast Buildup of a Fano Resonance in the Time Domain, Science 354, 738 (2016).

[38] V. Gruson, L. Barreau, Á. Jiménez-Galan, F. Risoud, J. Caillat, A. Maquet, B. Carré, F. Lepetit, J. F. Hergott, T. Ruchon, L. Argenti, R. Taïeb, F. Martín, and P. Salières, Attosecond Dynamics through a Fano Resonance: Monitoring the Birth of a Photoelectron, Science 354, 734 (2016).

[39] S. Beaulieu, A. Comby, A. Clergerie, J. Caillat, D. Descamps, N. Dudovich, B. Fabre, R. Géneaux, F. Légaré, S. Petit, B. Pons, G. Porat, T. Ruchon, R. Taïeb, V. Blanchet, and Y. Mairesse, Attosecond-Resolved Photoionization of Chiral Molecules, Science 358, 1288 (2017).

[40] L. Cattaneo, J. Vos, R. Y. Bello, A. Palacios, S. Heuser, L. Pedrelli, M. Lucchini, C. Cirelli, F. Martín, and U. Keller, Attosecond Coupled Electron and Nuclear Dynamics in Dissociative Ionization of $\mathrm{H}_{2}$, Nat. Phys. 14, 733 (2018).

[41] S. Biswas, B. Förg, L. Ortmann, J. Schötz, W. Schweinberger, T. Zimmermann, L. Pi, D. Baykusheva, H. A. Masood, I. Liontos, A. M. Kamal, N. G. Kling, A. F. Alharbi, M. Alharbi, A. M. Azzeer, G. Hartmann, H. J. Wörner, A. S. Landsman, and M. F. Kling, Probing Molecular Environment through Photoemission Delays, Nat. Phys. 16, 778 (2020).

[42] D. Trabert, S. Brennecke, K. Fehre, N. Anders, A. Geyer, S. Grundmann, M. S. Schöffler, L. P. H. Schmidt, T. Jahnke, R. Dörner, M. Kunitski, and S. Eckart, Angular Dependence of the Wigner Time Delay upon Tunnel Ionization of $\mathrm{H}_{2}$, Nat. Commun. 12, 1697 (2021).

[43] X. Gong, S. Heck, D. Jelivona, C. Perry, K. Zinchenko, and H. J. Wörner, Attosecond Spectroscopy of Size-Resolved Water Clusters, arXiv:2106.09459.

[44] I. Jordan, M. Huppert, D. Rattenbacher, M. Peper, D. Jelovina, C. Perry, A. V. Conta, A. Schild, H. J. Wörner, and M. Carlo, Attosecond Spectroscopy of Liquid Water, Science 369, 974 (2020).

[45] A. L. Cavalieri, N. Muller, T. Uphues, V. S. Yakovlev, A. Baltuska, B. Horvath, B. Schmidt, L. Blumel, R. Holzwarth, S. Hendel, M. Drescher, U. Kleineberg, P. M. Echenique, R. Kienberger, F. Krausz, and U. Heinzmann, Attosecond Spectroscopy in Condensed Matter, Nature (London) 449 , 1029 (2007).

[46] Z. Tao, C. Chen, T. Szilvási, M. Keller, M. Mavrikakis, H. Kapteyn, and M. Murnane, Direct Time-Domain Observation of Attosecond Final-State Lifetimes in Photoemission from Solids, Science 353, 62 (2016).

[47] M. Lucchini, S. A. Sato, A. Ludwig, J. Herrmann, M. Volkov, L. Kasmi, Y. Shinohara, K. Yabana, L. Gallmann, and U. Keller, Attosecond Dynamical Franz-Keldysh Effect in Polycrystalline Diamond, Science 353, 916 (2016).

[48] M. Ossiander, J. Riemensberger, S. Neppl, M. Mittermair, M. Schäffer, A. Duensing, M. S. Wagner, R. Heider, M. Wurzer, M. Gerl, M. Schnitzenbaumer, J. V. Barth, F. Libisch, C. Lemell, J. Burgdörfer, P. Feulner, and R. Kienberger, Absolute Timing of the Photoelectric Effect, Nature (London) 561, 374 (2018).

[49] T. Popmintchev, M. C. Chen, P. Arpin, M. M. Murnane, and H.C. Kapteyn, The Attosecond Nonlinear Optics of Bright Coherent X-Ray Generation, Nat. Photonics 4, 822 (2010).
[50] G. Saathoff, L. Miaja-Avila, M. Aeschlimann, M. M. Murnane, and H. C. Kapteyn, Laser-Assisted Photoemission from Surfaces, Phys. Rev. A 77, 022903 (2008).

[51] R. N. Zare, Photoejection Dynamics, Mol. Photochem. 4, 1 (1972), https://web.stanford.edu/group/Zarelab/publinks/ zarepub60.pdf.

[52] J. M. Dahlström, D. Guénot, K. Klünder, M. Gisselbrecht, J. Mauritsson, A. L'Huillier, A. Maquet, and R. Taïeb, Theory of Attosecond Delays in Laser-Assisted Photoionization, Chem. Phys. 414, 53 (2013).

[53] A. S. Kheifets, A. W. Bray, and I. Bray, Attosecond Time Delay in Photoemission and Electron Scattering near Threshold, Phys. Rev. Lett. 117, 143202 (2016).

[54] S. Heuser, Á. J. Galán, C. Cirelli, C. Marante, M. Sabbar, R. Boge, M. Lucchini, L. Gallmann, I. Ivanov, A. S. Kheifets, J. M. Dahlström, E. Lindroth, L. Argenti, F. Martín, and U. Keller, Angular Dependence of Photoemission Time Delay in Helium, Phys. Rev. A 94, 063409 (2016).

[55] M. Ossiander, F. Siegrist, V. Shirvanyan, R. Pazourek, A. Sommer, T. Latka, A. Guggenmos, S. Nagele, J. Feist, J. Burgdörfer, R. Kienberger, and M. Schultze, Attosecond Correlation Dynamics, Nat. Phys. 13, 280 (2017).

[56] J. Fuchs, N. Douguet, S. Donsa, F. Martin, J. Burgdorfer, L. Argenti, L. Cattaneo, and U. Keller, Time Delays from One-Photon Transitions in the Continuum, Optica 7, 154 (2020).

[57] P. Hockett, E. Frumker, D. M. Villeneuve, and P. B. Corkum, Time Delay in Molecular Photoionization, J. Phys. B 49, 095602 (2016).

[58] D. Baykusheva and H. J. Wörner, Theory of Attosecond Delays in Molecular Photoionization, J. Chem. Phys. 146, 124306 (2017).

[59] R. R. Lucchese and F. A. Gianturco, One-Electron Resonances in Electron Scattering from Polyatomic Molecules, Int. Rev. Phys. Chem. 15, 429 (1996).

[60] A. P. Natalense and R. R. Lucchese, Cross Section and Asymmetry Parameter Calculation for Sulfur 1s Photoionization of $\mathrm{SF}_{6}$, J. Chem. Phys. 111, 5344 (1999).

[61] F. Martin, P. D. Burrow, Z. Cai, P. Cloutier, D. Hunting, and L. Sanche, DNA Strand Breaks Induced by 0-4 eV Electrons: The Role of Shape Resonances, Phys. Rev. Lett. 93, 068101 (2004).

[62] N. Poccia, A. Ansuini, and A. Bianconi, Far from Equilibrium Percolation, Stochastic and Shape Resonances in the Physics of Life, Int. J. Mol. Sci. 12, 6810 (2011).

[63] A. McPherson, G. Gibson, H. Jara, U. Johann, T. S. Luk, I. A. McIntyre, K. Boyer, and C. K. Rhodes, Studies of Multiphoton Production of Vacuum-Ultraviolet Radiation in the Rare Gases, J. Opt. Soc. Am. B 4, 595 (1987).

[64] P. B. Corkum, Plasma Perspective on Strong Field Multiphoton Ionization, Phys. Rev. Lett. 71, 1994 (1993).

[65] M. Chini, H. Mashiko, H. Wang, S. Chen, C. Yun, S. Scott, S. Gilbertson, and Z. Chang, Delay Control in Attosecond Pump-Probe Experiments, Opt. Express 17, 21459 (2009).

[66] Y. Mairesse and F. Quéré, Frequency-Resolved Optical Gating for Complete Reconstruction of Attosecond Bursts, Phys. Rev. A 71, 011401(R) (2005).

[67] J. Gagnon, E. Goulielmakis, and V. S. Yakovlev, The Accurate FROG Characterization of Attosecond Pulses from Streaking Measurements, Appl. Phys. B 92, 25 (2008). 
[68] I. Jordan and H. J. Wörner, Extracting Attosecond Delays from Spectrally Overlapping Interferograms, J. Opt. 20, 024013 (2018).

[69] I. Jordan, M. Huppert, D. Rattenbacher, M. Peper, D. Jelovina, C. Perry, A. V. Conta, A. Schild, and H. J. Wörner, Attosecond Spectroscopy of Liquid Water, Science 369, 974 (2020).

[70] C. H. Zhang and U. Thumm, Electron-Ion Interaction Effects in Attosecond Time-Resolved Photoelectron Spectra, Phys. Rev. A 82, 043405 (2010).

[71] J. M. Dahlström, T. Carette, and E. Lindroth, Diagrammatic Approach to Attosecond Delays in Photoionization, Phys. Rev. A 86, 061402(R) (2012).

[72] J. M. Dahlström, A. L'Huillier, and A. Maquet, Introduction to Attosecond Delays in Photoionization, J. Phys. B 45, 183001 (2012).

[73] O. Jagutzki, A. Cerezo, A. Czasch, R. Dörner, M. Hattaß, M. Huang, V. Mergel, U. Spillmann, K. Ullmann-Pfleger, T. Weber, H. Schmidt-Böcking, and G. D. Smith, Multiple Hit Readout of a Microchannel Plate Detector with a ThreeLayer Delay-Line Anode, IEEE Trans. Nucl. Sci. 49, 2477 (2002).

[74] J. Ullrich, R. Moshammer, A. Dorn, R. Dörner, L. P. H. Schmidt, and H. Schmidt-Böcking, Recoil-Ion and Electron Momentum Spectroscopy: Reaction-Microscopes, Rep. Prog. Phys. 66, 1463 (2003).

[75] M. Lebech, J. C. Houver, and D. Dowek, Valence and Inner-Valence Shell Dissociative Photoionization of $\mathrm{CO}$ in the 26-33 eV Range. I. Ion-Electron Kinetic Energy Correlation and Laboratory Frame Photoemission, J. Chem. Phys. 130, 194307 (2009).

[76] M. Lebech, J. C. Houver, G. Raseev, A. S. Dos Santos, D. Dowek, and R. R. Lucchese, Valence and Inner-Valence Shell Dissociative Photoionization of $\mathrm{CO}$ in the 26-33 eV Range. II. Molecular-Frame and Recoil-Frame Photoelectron Angular Distributions, J. Chem. Phys. 136, 094303 (2012).
[77] X. Gong, M. Kunitski, L. P. H. Schmidt, T. Jahnke, A. Czasch, R. Dörner, and J. Wu, Simultaneous Probing of Geometry and Electronic Orbital of ArCO by CoulombExplosion Imaging and Angle-Dependent Tunneling Rates, Phys. Rev. A 88, 013422 (2013).

[78] G. Prümper, H. Fukuzawa, D. Rolles, K. Sakai, K. C. Prince, J. R. Harries, Y. Tamenori, N. Berrah, and K. Ueda, Is CO Carbon KVV Auger Electron Emission Affected by the Photoelectron?, Phys. Rev. Lett. 101, 233202 (2008).

[79] H. J. Werner, P. J. Knowles, G. Knizia, F. R. Manby, and M. Schütz, Molpro: A General-Purpose Quantum Chemistry Program Package, Comput. Mol. Sci. 2, 242 (2012).

[80] H. J. Werner, P. J. Knowles, F. R. Manby, J. A. Black, K. Doll, A. Heßelmann, D. Kats, A. Köhn, T. Korona, D. A. Kreplin, Q. Ma, T. F. Miller, A. Mitrushchenkov, K. A. Peterson, I. Polyak, G. Rauhut, and M. Sibaev, The Molpro Quantum Chemistry Package, J. Chem. Phys. 152, 144107 (2020).

[81] H. J. Werner, P. J. Knowles, G. Knizia, F. R. Manby, M. Schütz et al., MOLPRO 2019, a package of ab initio programs, see https://www.molpro.net.

[82] F. A. Gianturco, R. R. Lucchese, and N. Sanna, Calculation of Low-Energy Elastic Cross Sections for Electron-CF4 Scattering, J. Chem. Phys. 100, 6464 (1994).

[83] X. J. Liu, N. A. Cherepkov, S. K. Semenov, V. Kimberg, F. Gel'mukhanov, G. Prümper, T. Lischke, T. Tanaka, M. Hoshino, H. Tanaka, and K. Ueda, Young's Double-Slit Experiment Using Core-Level Photoemission from $N$ 2: Revisiting Cohen-Fano's Two-Centre Interference Phenomenon, J. Phys. B 39, 4801 (2006).

[84] X. J. Liu, G. Prümper, F. Gel'mukhanov, N. A. Cherepkov, H. Tanaka, and K. Ueda, Young's Double-Slit Experiment Using Two-Center Core-Level Photoemission: Photoelectron Scattering Effects, J. Electron Spectrosc. Relat. Phenom. 156-158, 73 (2007).

[85] B. Grieves and D. Dunn, Calculation of Continuum Wave Functions, Comput. Phys. Commun. 77, 313 (1993). 